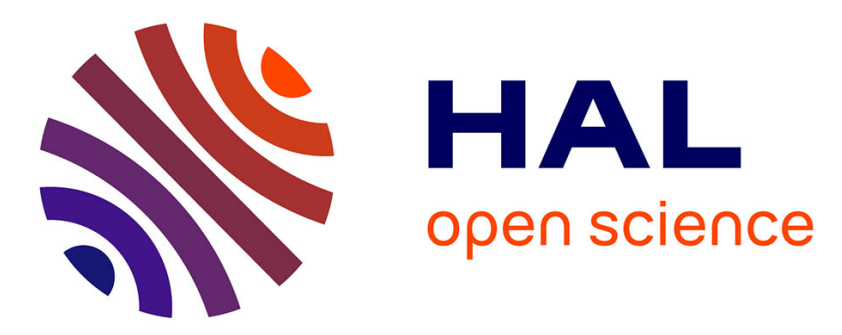

\title{
A selective mass scaling method for shear wave propagation analyses in nearly incompressible materials
}

\author{
W. Ye, A. Bel-Brunon, S. Catheline, M. Rochette, Alain Combescure
}

\section{To cite this version:}

W. Ye, A. Bel-Brunon, S. Catheline, M. Rochette, Alain Combescure. A selective mass scaling method for shear wave propagation analyses in nearly incompressible materials. International Journal for Numerical Methods in Engineering, 2017, 109 (2), pp.155 - 173. 10.1002/nme.5276 . hal-01864365

\section{HAL Id: hal-01864365 https://hal.science/hal-01864365}

Submitted on 29 Aug 2018

HAL is a multi-disciplinary open access archive for the deposit and dissemination of scientific research documents, whether they are published or not. The documents may come from teaching and research institutions in France or abroad, or from public or private research centers.
L'archive ouverte pluridisciplinaire HAL, est destinée au dépôt et à la diffusion de documents scientifiques de niveau recherche, publiés ou non, émanant des établissements d'enseignement et de recherche français ou étrangers, des laboratoires publics ou privés. 


\begin{abstract}
(1)

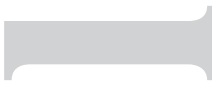

A selective mass scaling method for shear wave propagation analyses in nearly incompressible materials
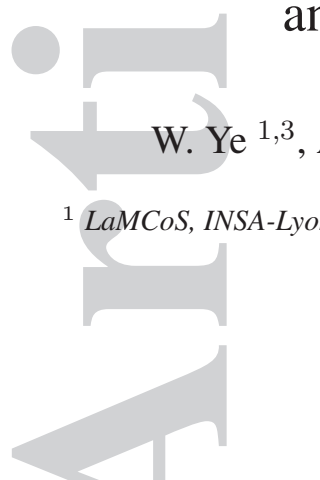

A. Bel-Brunon ${ }^{1}$, S. Catheline ${ }^{2}$, M. Rochette $^{3}$, A. Combescure ${ }^{1 *}$

, Université de Lyon, CNRS UMR 5259, France ${ }^{2}$ INSERM LabTAU Unit 1032, Université de Lyon, France ${ }^{3}$ ANSYS France, Montigny-le-Bretonneux, France

This paper describes a selective mass scaling (SMS) method which is designed for the analysis of wave propagation problems in nearly incompressible materials. The incompressibility of materials leads to a high value of the compressional wave speed which makes the time step extremely small in explicit time integration method. The proposed SMS method selects the eigenfrequencies related to volumetric deformation modes to decrease them, while it keeps the shear eigenmodes unchanged. This makes the time step no longer limited by the compressional wave speed but by the shear wave speed. A significant reduction of CPU time is obtained with a good accuracy for transient problems in small strains on free or largely prestressed media.
\end{abstract}

Copyright (c) 2016 John Wiley \& Sons, Ltd.

Received...

KEY WORDS: incompressible materials; wave propagation; selective mass scaling; explicit dynamic

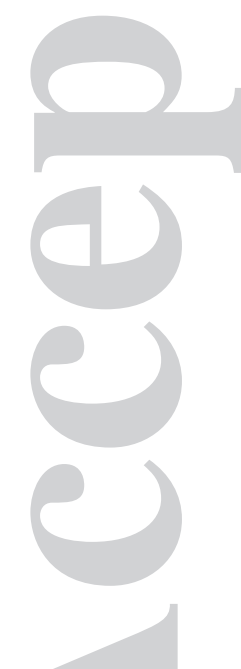

*Correspondence to: alain.combescure@ insa-lyon.fr

This article has been accepted for publication and undergone full peer review but has not been through the copyediting, typesetting, pagination and proofreading process, which may lead to differences between this version and the Version of Record. Please cite this article as doi: $10.1002 / \mathrm{nme} .5276$ 


\section{INTRODUCTION}

More and more finite element analysis (FEA) are used for surgical simulations [1, 2] and biomechanical simulations [3]. Many of them are carried out in dynamic domain, some even need transient analysis. As a consequence, a fast and high-quality time integration tool is valuable. Explicit time integration has shown its good performance in bio-mechanical domain thanks to its robustness for complex nonlinear problems.

The main limitation of the explicit time integration method (typically the central difference scheme) is that the size of time step is limited, since the algorithm is only conditionally stable. For a system with no damping, the critical time step takes the form:

$$
\Delta t_{\text {crit }} \leq \frac{2}{\omega_{\max }}
$$

where $\omega_{\max }$ is the maximum frequency of the assembled mesh. This equation is usually replaced by the following simplified expression for engineering applications:

$$
\Delta t_{\text {crit }} \leq \frac{L_{\text {min }}^{e}}{c_{L}}, \quad c_{L}=\sqrt{\frac{K+\frac{4}{3} G}{\rho}}
$$

where $L_{\text {min }}^{e}$ is the characteristic length of the smallest element, $c_{L}$ the compressional wave speed in the solid, $K$ the bulk modulus, $G$ the shear modulus and $\rho$ the density. This equation means that the time step must be smaller than the time required by a compressional stress wave to cross the smallest element of the mesh. Hence the time step is controlled by the compressional wave speed. For bio-materials which are nearly incompressible, this leads to a very small time step.

One approach used to amplify the critical time step for explicit time integration is the selective mass scaling (SMS) method. The basic idea is to add mass to reduce eigenfrequencies in high frequency range while changing the lower ones as little as possible [4].

Some SMS methods focus on thin wall structures, see in $[5,6,7]$. In this case, the time step is limited by the small thickness, the selective mass scaling consists in increasing the masses associated with the local rotations. Olovsson et al.[5] propose an acceleration filter for solid elements in order to reduce the relative motion among the nodes in thickness direction. Cocchetti et al.[6, 7] consider solid-shell elements, the displacements of nodes are represented by a linear transformation on the middle plane, then mass is added on the "deformation" of middle plane to decrease the high frequencies. These methods keep the scaled mass matrix in lumped form. They have a good performance for finite rotation and deformation problems. However, these methods can not be used directly for general 3D structures.

Frías et al.[8] propose a method to choose global high eigenfrequencies range by the proper orthogonal decomposition (POD) method, the mass scaling is applied only on the high eigenfrequencies. This method also keeps the lumped mass matrix but a pre-calculation with normal time steps is required at the beginning of simulations to create the POD space.

A number of SMS methods are proposed for general utilization for which no pre-calculation is required. For example, stiffness proportional method is described in [4, 9]. This method is accurate in numerous applications and it preserves the eigenmodes: this can be analytically proven. This method is referred to as existing SMS method in the present article. Tkachuk and Bischoff [10]

This article is protected by copyright. All rights reserved. 
present different strategies to construct the scaled mass matrix, and they prove mathematically that accurate SMS methods should not distort the eigenmodes of the original systems, which are related to the eigenspace of stiffness matrix.

For the above mentioned methods $[4,9,10]$, the scaled mass matrix can not be lumped. This leads to a larger CPU time to get the accelerations, especially for non linear problems. One way to avoid this time consuming step is to directly construct the inverse mass matrix as presented in $[11,12]$. Tkachuk and Bischoff [11] propose a general method based on the Hamilton's principle: the method shows its accuracy for many problems, but the reduction of computational cost is limited (typically $50 \%$ ).

To our knowledge, all these SMS methods are developed for inertia dominant problems, but not for transient dynamic problems. One reason is that the optimal SMS requires the knowledge of mode distributions in the spectrum [10]. For transient dynamic problems, the response mode distribution depends on the loading type which may change a lot for each problem. On the other hand, the amplification of time step is normally not desired for these problems, because the simulation duration is always short and the number of output requests is not far from the number of time step.

However, wave propagation in incompressible material is specific. The incompressibility makes the compressional wave speed much faster than the shear wave speed (typically 3 orders of magnitude larger for the biological tissues), but hardly any energy transmits by compressional wave. In other words, almost all strain energy is contained in shear wave propagation. So the time step required to accurately integrate the global system is much larger than the stable time step which is limited by the compressional wave.

It is worthy to mention others approaches for dynamic problems for incompressible materials. Larhiri et al.[13] presented a fractional time-step integration method which treats the compressional wave through an implicit method but uses an explicit scheme for the shear wave. The stable time step then depends only on the shear wave speed. The method is then extended to Petrov-Galerkin formulation to enforce the stabilization $[14,15]$.

In present work, the SMS method is applied in such a way that the stable time step is driven by the shear wave speed and no more by the compressional wave speed. The shear eigenmodes and the volumetric eigenmodes are separated, the mass scaling is then applied only on the volumetric modes. Selective integration is used to control locking for simple elements. This method results in a mass matrix which can not be lumped. For geometrically linear problems, the resulting scaled mass matrix is inverted only once during the whole simulations. Unlike the implicit time integration methods, no iteration is needed to deal with the material non-linearity. Finally, a significant reduction of computational costs is obtained with a good accuracy.

This work is presented as follow. Section 2 describes the separation between the shear eigenmodes and volumetric eigenmode as well as the reduced/selective integration for quadrilateral and hexahedral elements. Sect. 3 recalls the existing SMS method, then the proposed method is described. A numerical validation is also included in this section. Some numerical applications are presented in Sect. 4, and the conclusions are given in Sect. 5.

This article is protected by copyright. All rights reserved. 


\section{SEPARATION OF SHEAR AND VOLUMETRIC EIGENMODES}

For a linear elastic isotropic material, the constitutive matrix $\mathbf{D}$ can be written by two independent parameters: $K$ for bulk modulus and $G$ for shear modulus. Then it can be divided into a deviatoric part and a volumetric part as follows:

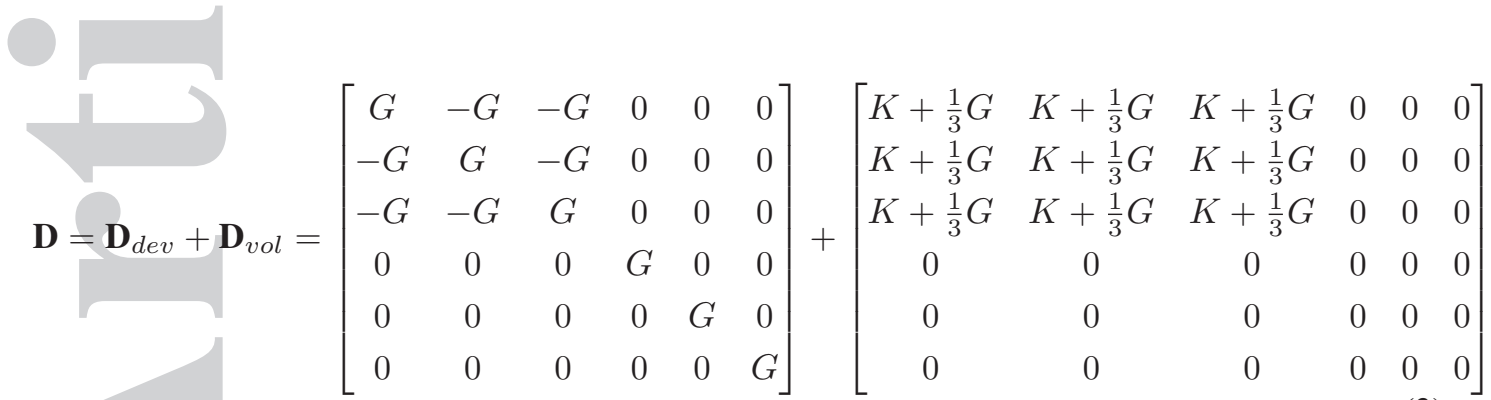

Notice that $\frac{1}{3} G$ appears in $\mathbf{D}_{v o l}$ which means that a pure shear deformation also leads pressure even if there is no volume change, this is known as the Kelvin effect [16]. However, this effect is only significant for large strain and it can be neglected for nearly incompressible materials for which $K$ is much larger than $G$.

Using above expression, the elemental stiffness matrix $\mathbf{K}^{e}$ can also be divided into two parts:

$$
\begin{aligned}
\mathbf{K}^{e} & =\mathbf{K}_{S}^{e}+\mathbf{K}_{L}^{e} \\
& =\int_{\Omega^{e}} \mathbf{B}^{\mathbf{T}} \mathbf{D}_{d e v} \mathbf{B} \mathrm{d} \Omega^{e}+\int_{\Omega^{e}} \mathbf{B}^{\mathbf{T}} \mathbf{D}_{v o l} \mathbf{B} \mathrm{d} \Omega^{e}
\end{aligned}
$$

where $\mathbf{B}$ is the displacement-strain operator, and $\Omega^{e}$ is the element integration domain. The two parts of stiffness matrix ( $\mathbf{K}_{S}^{e}$ and $\mathbf{K}_{L}^{e}$ ) may be integrated by different strategies.

$\mathbf{K}^{e}$ being a symmetric, positive-definite matrix, its spectral expression is:

$$
\mathbf{K}^{e}=\sum_{i=1}^{n d o f} \gamma_{i}^{e} \Psi_{i}^{e T} \Psi_{i}^{e}
$$

where $n d o f$ is the number of degrees of freedom of the element, $\gamma_{i}^{e}$ denotes the eigenvalues sorted in ascending order and $\Psi_{i}^{e}$ are the corresponding eigenvectors. In 2D (resp. 3D), there should be 3 (resp. 6) zeros eigenvalues correspondinh to the 3 (resp. 6) rigid body eigenmodes. It can be seen that these modes have no contribution on the stiffness. The others eigenmodes $\left(\gamma_{i}^{e} \neq 0\right)$ represent all possible deformations of an element. We will now show that they can be differentiated when specific integration strategies are employed.

Let us now focus on an ideal four-node square element with unit length side, the eigenvectors $\Psi_{i}^{e}$ and eigenvalues $\gamma_{i}^{e}$ can be calculated analytically. We consider the three possible integration strategies: the reduced integration (R.I.) which uses one Gauss points for both $\mathbf{K}_{S}^{e}$ and $\mathbf{K}_{L}^{e}$; the selective integration (S.I.) which uses four Gauss points for $\mathbf{K}_{S}^{e}$ but one point for $\mathbf{K}_{L}^{e}$; and the full integration (F.I.) which uses four points for both $\mathbf{K}_{S}^{e}$ and $\mathbf{K}_{L}^{e}$.

The results are displayed in Tab.I, each element has 8 modes (see in Fig.1): three rigid motion modes, two hourglass modes, two shear deformation modes and one volumetric deformation mode.

This article is protected by copyright. All rights reserved. 
The eight eigenvectors are all orthogonal to each other. We observe that the eigenvectors are the same for the three integration methods. In fact, they depend only on the shape of the element. Let us now consider the eigenvalues: the three integration methods give the same results for the rigid motions, shear modes and volumetric mode. The shear modes only depend on the shear modulus $G$, and the volumetric mode depends on both $K$ and $G$ but $K$ is dominant. The difference appears at hourglass modes. For reduced integration, the eigenvalues go to zero. It is known that this will lead spurious deformation in simulations if no stabilization is applied. For selective integration, the eigenvalues depend only on $G$, consequently, they will be considered as shear modes in the following. For full integration, the eigenvalues are a combination of $K$ and $G$. They can be neither seen as the volumetric modes nor as the shear modes. For nearly incompressible materials $(K \gg G)$, it produces locking problems.

To summarize, for reduced or selective integration, the deformation eigenmodes can be gathered in one group depending only on shear modulus and one group depending mainly on volumetric modulus. The conclusion can be extended for irregular elements, but no analytical solution is available. This is the premise to apply selective mass scaling only on the wanted mode, volumetric mode in this work. Similar decomposition can be found in $[17,18]$, where decomposition is realized modally.

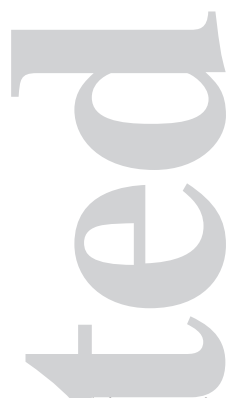

\section{SELECTIVE MASS SCALING METHOD}

In this section, we present the proposed SMS method which is designed to modify only the volumetric eigenmode with no change for shear eigenmodes. The method is then numerically validated by comparing the eigenvalues and eigenvectors between the original system and the scaled system for a model generated randomly.

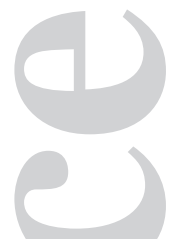

\subsection{General form of SMS}

Most SMS methods consist of defining the new global mass matrix $\overline{\mathbf{M}}$ by the expression

$$
\overline{\mathbf{M}}=\bigcup_{e} \overline{\mathbf{M}}^{e}, \quad \overline{\mathbf{M}}^{e}=\mathbf{M}^{e}+\boldsymbol{\lambda}^{e}
$$

where $\bigcup_{e}$ is the assembly operator over each element, $\overline{\mathbf{M}}^{e}$ is the elemental scaled mass matrix, $\mathbf{M}^{e}$ is the original mass matrix, which is often written in lumped form for the explicit time integration, and $\lambda^{e}$ is the added artificial mass. The objective of SMS methods is to find an appropriate $\lambda^{e}$ which reduces the high eigenfrequencies of the system while preserving the eigenmodes in lower frequency range.

This article is protected by copyright. All rights reserved. 
Now consider the finite element discretized problem having $n$ degrees of freedom, the solution at time $t+\Delta t$ computed with the explicit time integration scheme using the scaled mass matrix $\overline{\mathbf{M}}$ writes:

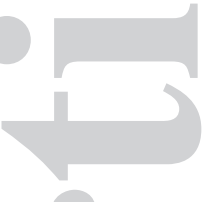

$$
\begin{aligned}
& { }^{t+\Delta t} \mathbf{U}={ }^{t} \mathbf{U}+\Delta t{ }^{t} \dot{\mathbf{U}}+\frac{1}{2} \Delta t^{2}{ }^{t} \ddot{\mathbf{U}} \\
& \overline{\mathbf{M}}^{t+\Delta t} \ddot{\mathbf{U}}={ }^{t+\Delta t} \mathbf{R} \\
& { }^{t+\Delta t} \dot{\mathbf{U}}={ }^{t} \dot{\mathbf{U}}+\frac{1}{2} \Delta t\left({ }^{t} \ddot{\mathbf{U}}+{ }^{t+\Delta t} \ddot{\mathbf{U}}\right)
\end{aligned}
$$

where $\mathbf{U}, \dot{\mathbf{U}}, \ddot{\mathbf{U}}$ represent the nodal displacements, velocities and accelerations respectively, $\mathbf{R}$ is the nodal residual force.

The change of solver $\mathbf{M}$ to $\overline{\mathbf{M}}$ leads to a change of stability limit. One can apply an amplification factor $\alpha$ to the usual critical time step.

$$
\Delta t=\alpha \Delta t_{\text {crit }}
$$

We shall see later how $\alpha$ can be estimated. It is important to note that the scaled mass matrix cannot be lumped, this would lead the Eq.7b to be computationally costly for large size problems. However, $\overline{\mathbf{M}}$ can be inverted only once at the beginning of simulations for geometrically linear problems. The examples in the next section will show that.

\subsection{The existing SMS method}

Stiffness proportional mass scaling is presented in [4]: it can be seen as the basic method of SMS family. $\lambda^{e}$ is chosen to be:

$$
\lambda^{e}=\beta \boldsymbol{K}^{e}, \quad \beta 0
$$

where $\mathbf{K}^{e}$ is the tangential stiffness matrix, and $\beta$ is a factor to be determined. The eigenfrequencies of the scaled system can be obtained analytically (see [4] for details):

$$
\bar{\omega}_{i}^{2}=\frac{\omega_{i}{ }^{2}}{1+\beta \omega_{i}{ }^{2}}, \quad i \in\{1, \ldots, n\}
$$

where $\omega_{i}$ represents the eigenfrequencies of the original system sorted in ascending order, and $\bar{\omega}_{i}$ represents the eigenfrequencies for the scaled system. It can be observed that the high values of the eigenfrequencies decrease much more than the lower ones while the rigid motion modes are not affected (eigenfrequency equals 0 ) .

By applying the above expression for the maximal frequency $\omega_{\max }$, and by considering the Eq.1, the following expression is obtained to estimate the factor $\beta$ :

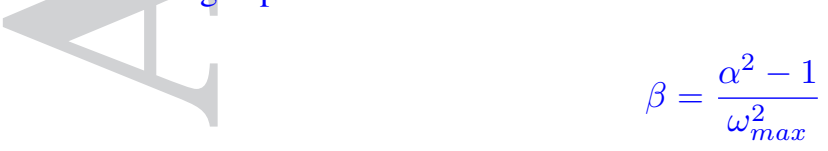

Before going into the next section, let us take another way to look the existing method using the conclusion in section 2 . The shear eigenmodes and the volumetric eigenmode are totally separated in

This article is protected by copyright. All rights reserved. 
selective/reduced integration, and the volumetric part has only one eigenmode. Eq.9 can be written in spectral form:

$$
\lambda^{e}=\beta\left(\gamma_{L}^{e} \Psi_{L}^{e T} \Psi_{L}^{e}+\sum_{i=1}^{n_{S}} \gamma_{S i}^{e} \Psi_{S i}^{e T} \Psi_{S i}^{e}\right)
$$

where $\gamma_{L}^{e}$ and $\Psi_{L}^{e}$ are the eigenvalue and eigenvector of volumetric mode, $\gamma_{S}^{e}$ and $\Psi_{S}^{e}$ are the eigenvalues and eigenvectors of shear modes ( $n_{S}$ is the number of shear modes).

For quadrilateral element, $n_{S}=2$ with reduced integration and $n_{S}=4$ with selective integration. For hexahedral 3D element, $n_{S}=6$ with reduced integration and $n_{S}=12$ with selective integration. It should be mentioned that the above form is referred to as the local spectral SMS method in [10].

The above expression (Eq.12) leads us to observe that the existing SMS method "selects" to scale down all the deformation modes (both shear modes and volumetric mode), but not the rigid body modes: this is why it works well for inertia dominant problems. However, for wave propagation problems, the deformation modes have the same importance as the rigid motion modes, this method is not so well suited.

\subsection{The proposed SMS method}

In the simulations of nearly incompressible materials, the volumetric mode contains very little energy but leads to spurious high frequencies. For this reason, it is proposed to scale only the volumetric eigenmode of the stiffness matrix, which writes:

$$
\lambda^{e}=\beta \gamma_{L}^{e} \Psi_{L}^{e T} \Psi_{L}^{e}
$$

Using this expression requires calculating the volumetric eigenmode for each element which is time consuming. For the implementation simplicity, we use $\boldsymbol{\lambda}^{e}=\beta \mathbf{K}_{L}^{e}$ ( $\mathbf{K}_{L}^{e}$ should be integrated with one Gauss point).

This method can be regarded as an extension of the existing SMS method, the same equation (Eq.11) can be used to determine $\beta$. Theoretically, choosing $\beta$ large enough makes volumetric eigenfrequencies very small. However, as the proposed method does not affect any shear eigenmode, the stable time step is still limited by the maximum frequency of the shear deformation mode, denoted by $\omega_{S, \max }$. For this reason, the amplification factor $\alpha$ must verify:

$$
1 \alpha \frac{\omega_{\max }}{\omega_{S, \max }}
$$

The above equation (Eq.14) requires the knowledge of the eigenfrequencies for the whole model. This ratio of eigenfrequencies can be simply estimated using the two wave speeds: $\frac{\omega_{\max }}{\omega_{S, \max }} \simeq \frac{C_{L}}{C_{S}}$. By using the maximum admissible value of $\alpha$, we can say that the time step is no longer limited by the compressional wave speed, but the shear wave speed. For nearly incompressible materials, the two wave speed have a huge difference, $\alpha$ can be very large. In practice, as usual in the pure explicit method, a safety coefficient is used to take account for mesh distortion and approximation of eigenfrequencies, which leads $\alpha=0.9 \frac{C_{L}}{C_{S}}$

This article is protected by copyright. All rights reserved. 


\subsection{Validation}

In this section, we aim to validate the preservation property of proposed SMS method. The characteristic equations for the undamped linear systems are:

- For the original system:

$$
\left(\mathbf{K}-\omega^{2} \mathbf{M}\right) \Phi=\mathbf{0}
$$

-For the scaled system:

$$
\left(\mathbf{K}-\bar{\omega}^{2} \overline{\mathbf{M}}\right) \bar{\Phi}=\mathbf{0}
$$

where $\omega_{i}$ and $\bar{\omega}_{i}$ have been introduced in Section 3.2, $\Phi_{i}$ and $\bar{\Phi}_{i}$ represent the corresponding eigenvectors.

As pointed out previously $[4,10]$, the premises to achieve accurate solutions with SMS method are the preservation or small variation of eigenmode values and vectors. The preservation is not necessarily applying for all eigenmodes, but must be assured for the relevant modes which have high energy content. In real applications, due to the complexity of structures and various types of loads, the important eigenmodes are difficult to know a priori. However, nearly incompressible materials have a specific property, because of the huge difference between bulk modulus and shear modulus: the structure deforms barely in the volumetric way, almost all the potential energy stores in shear deformation modes. This is quite universal for all loading.

In order to examine the preservation property of the proposed method, only the eigenmodes related to shear deformations should be considered. A test model of 50 degrees of freedom is presented in Fig.2. Considering a 2D plate with $4 \times 4$ elements, the nodes position is given in a random way. The material parameters are shown in the figure, they correspond to strong incompressibility, the ratio of the compressional wave speed to the shear wave speed equals 100 . Accordingly, the amplification factor $\alpha$ is set to 90 to ensure stability of the explicit time stepping. Hence, $\beta=1.54 \times 10^{-7}$ (Eq.11).

The reasons why an irregular mesh is used are:

- The sensitivity to mesh distortions is checked;

- No symmetric eigenmode will be produced (equal eigenvalue).

The mass matrix $\mathbf{M}$ is lumped, and the stiffness matrix $\mathbf{K}$ is formed by using selective integration strategy, with plane strain hypothesis.

\subsubsection{Preservation of eigenvalues}

Eigenvalues of the test model are calculated respectively for the original unscaled system, the system scaled by the existing SMS method, and the system scaled by the proposed SMS method. The results are displayed in Fig.3. The first three eigenvalues which equal 0 are not shown, they correspond to 3 rigid motion modes.

Fig.3(a) shows that an important jump appears at the $35 t h$ mode for the original system (red points): the eigenvalues experience a huge increase from this point. Herein, the eigenmodes from $4-34$ correspond to shear deformation modes, while modes $35-50$ correspond to volumetric deformation ones. Notice that there are 16 volumetric modes: this is the number of elements of the system.

\section{Remark:}

This article is protected by copyright. All rights reserved. 
When one replaces $\mathbf{K}$ by $\mathbf{K}_{L}$ (the volumetric stiffness) in equation Eq.15, only the high eigenvalues $(35-50$ modes $)$ are found. While $\mathbf{K}_{S}$ is used, all eigenvalues are small. This observation confirms the deduction above.

More importantly, the figure (Fig.3(a)) shows that the volumetric eigenvalues obtained by both SMS methods decrease significantly (up to the same value of shear eigenvalues), while the shear eigenvalues remain unchanged. A zoom is presented in Fig.3(b): the eigenvalues for the original system and for the system scaled by the proposed SMS method are the same. However, the existing SMS method does not match so well. For instance, the eigenvalues are decreased of about $50 \%$ than the original ones. This will introduce notable errors in simulation of shear wave propagations.

\subsubsection{Preservation of eigenvectors}

As eigenvectors are also affected, a criterion is needed to quantify the eigenvectors distortion. Thus, $\varphi_{i}$ which represents the distortion angle between original system and scaled system for $i t h$ mode is introduced [10]:

$$
\varphi_{i}=\arccos \left(\frac{\bar{\Phi}_{i} \Phi_{i}}{\left|\bar{\Phi}_{i}\right|\left|\Phi_{i}\right|}\right)
$$

Fig.4 shows the values of distortion angles for all eigenmodes of the test model. For shear eigenmode vectors, the two SMS methods have same performance, no distortion is observed. For volumetric eigenvectors, the existing SMS keeps the mode shapes while the proposed SMS leads to important distortions. The result about the existing SMS method is coincident with the analytical solution in $[4,9]$. However, it must be mentioned that these distortions have no significant effect in the solutions, because volumetric modes contain very little energy. Examples will emphasize this observation in the next section.

In Fig. 5 and 6, three shear eigenvectors and three volumetric eigenvectors are chosen to illustrate the distortion. For shear eigenvectors, the eigenvectors for the three systems are identical. For volumetric eigenvectors, the modes of the existing SMS method are the same as those of the original system, while the proposed SMS method leads to distortions.

We can conclude from the presented test model, that both SMS methods decrease high eigenfrequencies, which means a larger stable time step can be obtained. The proposed SMS method keeps both shear eigenvalues and eigenvectors with a good accuracy, but does not preserve the volumetric eigenvalues and eigenvectors, which barely influences in the simulations. However, the

existing SMS will lead to inaccurate results in simulation of shear dominated transient problems because the shear eigenvalues are not well preserved.

\section{NUMERICAL EXAMPLES}

To illustrate the performance of the proposed method, several examples of wave propagation problems with nearly incompressible elastic materials are presented. The objective is to show that the proposed SMS method improves greatly the computational speed when compared to the pure explicit one, while the accuracy is still guaranteed.

This article is protected by copyright. All rights reserved. 
All simulations are carried out by our Finite Element code implemented in Fortran. For the SMS methods, the algorithm PARDISO [19] is used as the inverse matrix solver.

Selective integration is used in all the examples. Reduced integration is also available, but the hourglass modes should be carefully controlled.

\subsection{Cantilever beam}

The first example is a cantilever beam problem, the model is described in Fig.7. The initial velocity $V_{0}=10 \mathrm{~mm} / \mathrm{s}$ is applied in $x$ direction for all the nodes except the left end. The simulation time lasts for $0.01 \mathrm{~s}$.

The mesh consists of 3072 regular cubic elements with a side length of $l_{e}=0.125 \mathrm{~mm}$. The time step is estimated by $\Delta t=0.8 \frac{l_{e}}{C_{L}}$ for the pure explicit simulations, and $\Delta t=0.8 \frac{l_{e}}{C_{S}}$ for the proposed SMS simulations. The material parameters are: the shear modulus $G=0.001 M P a$, the density $\rho=1000 \mathrm{~kg} / \mathrm{m}^{3}$, so the shear wave speed is fixed to $C_{S}=1 \mathrm{~m} / \mathrm{s}$. The Poisson's coefficients are given different values to obtain different levels of incompressibility, see Tab. II. The ratio of the compressional wave speed to the shear wave speed increases while the Poisson's coefficient approaches to 0.5 . Consequently, the explicit method needs more time steps to complete the simulations. However, for SMS method, the stable time step is kept constant. The CPU cost of the simulation is therefore even more decreased as the ratio $C_{L} / C_{S}$ gets higher. The time saving gets remarkable for $C_{L} / C_{S}$ reaching 1000 , which is a typical value for biological tissues.

The displacement results along A-A' point are shown in Fig.8, the results obtained by the explicit method are displayed by lines, and the results obtained by the proposed SMS method are displayed by different marks. For different Poisson's coefficients, the results are presented by different colors at different time points. The explicit method and the SMS method give exactly the same results. We would like to point out here that the problem being not in transient dynamic (wave propagation) range, the use of the extreme Poisson's coefficient does not have significant effect on simulation results (at the same time point, using different Poisson's coefficients yields almost the same curve). But the objective of this example is to show the computational performance of the proposed SMS method. We experience that the proposed method is much more efficient in applications of highly incompressible materials.

\subsection{Circular shear wave propagation}

The above example shows that in the proposed method, the time step is not controlled by the compressional wave speed anymore, but by the shear wave speed. However, it is also important to consider the influence of the frequency of external load.

A shear wave propagation problem is considered in plane strain hypotheses, the model is shown in Fig.9(a). The shear modulus is set to $G=0.001 M P a$, Poisson's coefficient $\nu=0.49995$ and the density $\rho=1000 \mathrm{~kg} / \mathrm{m}^{3}$. These parameters yield a compressional wave speed $C_{L}=100 \mathrm{~m} / \mathrm{s}$, and a shear wave speed $C_{S}=1 \mathrm{~m} / \mathrm{s}$. Line forces are applied at the center zone defined by Ricker wavelet function:

$$
f(t)=A\left(1-2 \pi^{2} f^{2}\left(t-t_{0}\right)^{2}\right) \exp \left(-\pi^{2} f^{2}\left(t-t_{0}\right)^{2}\right)
$$

with the amplitude $A=0.0001 \mathrm{~N}$, the frequency $f=300 \mathrm{~Hz}$ and $t_{0}=0.003 \mathrm{~s}$. In this example, the critical time step for classical explicit method is limited to $5 \times 10^{-6} \mathrm{~s}$. By applying the selective 
mass scaling, the maximal amplification of time step can be theoretically $\alpha=100$, which leads

to $\Delta t=5 \times 10^{-4} \mathrm{~s}$. However, the time step should also be smooth enough to approximate the load function. Finally, we choose to set $\alpha=30$ and $\alpha=60$, the time steps are $1.5 \times 10^{-4} s$ and $3 \times 10^{-4} \mathrm{~s}$ accordingly. The load function with $\Delta t=3 \times 10^{-4} \mathrm{~s}$ is plotted in Fig.10(b), we note that its shape is well approximated, yet not very accurate. Its effect on the simulation results is presented later.

Fig.10 shows the velocity and the shear stress field at the end of the simulation $(t=0.03 \mathrm{~s})$. The result is obtained by the proposed SMS method with $\alpha=30$. We observe the shear wave front in a circular form reaching the edge of the plate. The results obtained by the pure explicit method are not presented here, because they are the same as Fig.10. More detailed comparisons are displayed through the measuring points.

Fig.11 shows the displacement history for three measuring points A, B and C. Notice that all the results present post oscillations because the used methods are all explicit. Generally, the proposed SMS with $\alpha=30$ (green) gives result which is close to the pure explicit method (red). For results obtained by SMS with $\alpha=60$ (blue), only the shear wave front is well described. For example, at point $\mathrm{A}$ and $\mathrm{B}$, the blue curves are strongly dephased compared to the reference (red), while the green ones present a much better coherence. At point $\mathrm{C}$, which is far from the load, we note that the wave front just arrives, and the amplitude of blue curve is superior than the other two.

Fig. 12 shows the vertical velocity distribution along O-O' at three different times. We observe that the post oscillations are more serious for the velocities. At the beginning of the simulation ( $t=0.006 s$ ), three results are the same. The result with $\alpha=60$ (blue) leads much more distortion as the wave propagates. A similar conclusion can be done: SMS with $\alpha=30$ yields much better results than $\alpha=60$.

As presented earlier, choosing a high scaling factor $\alpha$ induces more errors in results, even though the algorithm is still stable. Moreover, the computational speed is not necessarily improved. In this example, the explicit method takes $189 \mathrm{~s}$, the SMS with $\alpha=30$ takes $17 \mathrm{~s}$, and the SMS with $\alpha=60$ takes $13 \mathrm{~s}$. Consequently, when using SMS method, the amplification factor of time step should be well considered in order to improve the computational speed while maintaining the accuracy. In this example, the loading must be described with a sufficient number of points which limits $\alpha$ to 30 .

\subsection{Planar shear wave propagation in heterogeneous media}

The two previous examples can also be performed by the existing SMS method. Its efficiency is the same as the proposed SMS method, however, because of the poor preservation of eigenvalues for shear deformation modes, the accuracy of simulation is not so good. A model of planar shear wave propagation in a heterogeneous medium is now presented to illustrate this point.

We consider a square plate in plane strain which is constituted by two linear elastic materials, as shown in Fig.13. The mesh has 3600 square elements. The material parameters are summarized in Tab.III, in which $G$ is the shear modulus, $\nu$ is the Poisson's coefficient and $C_{L}$ and $C_{S}$ represent the compressional wave speed and the shear wave speed respectively.

An initial velocity of $10 \mathrm{~mm} / \mathrm{s}$ is applied at the top side and released after $0.0008 \mathrm{~s}$ (see Fig.13). The simulation time is set to $0.16 s$ so that the shear wave travels through the whole model. Note that for pure explicit method, the time step is controlled by the compressional wave speed of material I, which is $0.8 \times 10^{-5} \mathrm{~s}$. While using SMS method (both existing and proposed), the time step 
raises up to $0.8 \times 10^{-3} s$ just by considering the faster shear wave speed in the two materials. For computational speed, the pure explicit method costs $221 \mathrm{~s}$, and the two SMS methods cost around $10 \mathrm{~s}$.

Fig.14, 15 show the displacement and velocity distribution along A-A'. The advancement of shear wave front is clearly observed at different time. The proposed SMS method (green) presents the results which are very close to the explicit method (red), for both displacements and velocities. However, the existing SMS results (blue) is not accurate.

Fig.16 and 17 show the displacement and the velocity history at three measuring points. The difference between the two SMS methods is quite clear, the proposed method results is more accurate than the existing SMS method.

In Fig.18, the shear stress contour plot are compared at times $t=0.064 \mathrm{~s}$ and $t=0.16 \mathrm{~s}$. The time points are chosen so that the shear wave front reaches the heterogeneous part, so one can distinguish the heterogeneity position by stress distribution. The explicit method and the proposed SMS method show the two heterogeneous parts as expected. The existing SMS method gives unfaithful results. Particularly, the second heterogeneous block is not visible in Fig.18(b).

\subsection{Shear wave propagation in a pre-stressed medium}

This example could be considered as an application of a medical imaging technology named elastography [20]. We consider wave propagation in a pre-stressed medium: a dynamic load is applied on a statically pre-deformed state. Since the pre-stressing load is much higher than the dynamic load, the geometrical non-linearity in the dynamic step can be neglected, the problem is called small on big [21].

The model considered is described in Fig.19. As shown, due to symmetry, just a half domain is described. The static pressure is applied at the top with a ramp distribution, the maximum is set to $0.002 \mathrm{~N}$ and the minimum is zero. A Neo-Hookean hyper-elastic law is employed for the material, for which the elastic potential can be expressed in terms of the right Cauchy-Green tensor $\mathbf{C}$, the Jacobian $J$, and two constant as :

$$
W=C_{10}\left(J^{-2 / 3} \operatorname{tr}(\mathbf{C})-3\right)+\frac{K}{2}(J-1)^{2}
$$

The properties are chosen in the range of biological tissues, where $\rho=1000 \mathrm{~kg} / \mathrm{m}^{3}, C_{10}=$ $0.001 M P a, K=10 M P a$. These parameters lead to the speed of compressional wave $C_{L}=$ $100 \mathrm{~m} / \mathrm{s}$, and the shear wave speed $C_{s}=1.41 \mathrm{~m} / \mathrm{s}$ in small deformation.

First, the static simulation is carried out by classical Newton-Raphson algorithm, both material and geometrical non-linearities are considered. The results are shown in Fig.20, we observe the model deforms in the range of finite strain. Based on this state, a small sinusoidal load is then added at the top-left corner (see Fig.20): the vertical displacement with an amplitude $0.1 \mathrm{~mm}$, and a frequency of $100 \mathrm{~Hz}$ is imposed and released at $t=0.03 \mathrm{~s}$. The static pressure is still kept to avoid rebound. Dynamic simulation is carried out both by explicit method and proposed SMS method. During this simulation, we treat only material non-linearity but not geometrical non-linearity as the dynamic strain remains small.

The dynamic simulation lasts $0.05 \mathrm{~s}$. By considering the deformation of the mesh under the static load, the time step for the explicit method is set to $5 \times 10^{-6} \mathrm{~s}$, and for SMS method is $2.5 \times 10^{-4} \mathrm{~s}$

This article is protected by copyright. All rights reserved. 
which is 50 times larger than previous. For computational speed, the explicit method costs $110 \mathrm{~s}$ but the SMS method costs just $13 \mathrm{~s}$.

Fig.21 shows the dynamic displacement field at the end of the simulation. For both results, shear wave front (in the medium) and Rayleigh wave front (at the superior surface) are clearly distinguishable. No visible difference can be found on the figure. Then, it is important to note the amplitude is largely smaller than the static displacements field.

Fig.22, 23 show the displacement and the velocity history in time for three measuring points. We can observe that the explicit solution generates high frequency oscillations, especially for velocities. The SMS method has much less oscillations, this is caused by using the larger time step. However, the two results are very close. No significant difference is observed for average values, both for displacements and velocities.

The time evolution of energy is described in Fig. 24 . In Fig.24(a), the internal energy is divided into two parts: deviatoric part and volumetric part. We can observe that the deviatoric internal energy curve is the same for the two methods but not the volumetric one. However, it is important to note that the volumetric energy represents less than $3 \%$ of the whole internal energy. Fig.24(b) shows the two methods give very close kinetic energy evolution in time.

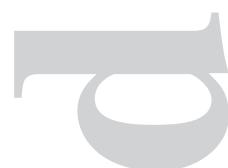

\section{CONCLUSION}

A selective mass scaling method based on volumetric and shear mode separation has been presented. The method aims at increasing the stable time step in explicit analyses for nearly incompressible materials. The analysis shows that the proposed method keeps exactly the eigenmodes related to the shear deformation, so the method is accurate for analysis of nearly incompressible materials in which shear deformation is dominant.

The method might be less interesting for inertia dominant problems, for which one can choose a smaller value of bulk modulus with nearly no effect on the dynamical response. However, for wave propagation analysis, keeping the real incompressibility is important. The proposed method is very useful in this domain to reduce the CPU time, for example, in case of analysis of wave propagation in biological soft tissues, such as elastography [22]. Moreover, the method was initially developed for biological materials which are often anisotropic. The anisotropy only applies to the deviatoric part [23]: this will result in a different expression of $\mathbf{D}_{d e v}$ (Eq.3), but nearly no change in $\mathbf{D}_{v o l}$ if $G \ll K$. The proposed method should then be applicable as it is.

Besides, the method is based on adding artificial terms to the mass matrix. The added term can not be lumped, which leads to a mass matrix which is no longer diagonal. As shown in examples, for linear geometrical problems, the scaled mass matrix is inverted only once. For nonlinear materials, no iteration is required in the explicit method. Moreover, it is known that the implicit methods lead to the pre-shock oscillations and the explicit methods lead to the post-shock oscillations, see in $[17,18]$. For wave propagation simulations, the latter case is more interesting. So the explicit time integration is really interesting in this context.

For nonlinear geometric problems, the scaled mass matrix has to be updated regularly, an iterative algorithm is available [24], but it is still heavy. Further work is still needed to improve that point.

This article is protected by copyright. All rights reserved. 
W. YE ET AL.

\section{ACKNOWLEDGEMENT}

The authors wish to thank the support of the CIFRE convention $\mathrm{N}^{\circ} 2013-1553$.

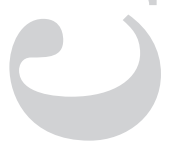

\section{REFERENCES}

1. Joldes GR, Wittek A, Miller K. Non-locking tetrahedral finite element for surgical simulation. Communications in Numerical Methods in Engineering 2009; 25(7):827-836.

2. Gindre J, Bel-Brunon A, Combescure A, Haigron P, Rochette M, Lucas A. Estimation of clinically relevant indicators for evar using patient-specific finite element simulation. Computer methods in biomechanics and biomedical engineering 2015; 18(sup1):1950-1951.

3. Ruan J, Khalil T, King AI. Dynamic response of the human head to impact by three-dimensional finite element analysis. Journal of Biomechanical Engineering 1994; 116(1):44-50.

4. Olovsson L, Simonsson K, Unosson M. Selective mass scaling for explicit finite element analyses. International Journal for Numerical Methods in Engineering 2005; 63(10):1436-1445.

5. Olovsson L, Unosson M, Simonsson K. Selective mass scaling for thin walled structures modeled with tri-linear solid elements. Computational Mechanics 2004; 34(2):134-136.

6. Cocchetti G, Pagani M, Perego U. Selective mass scaling and critical time-step estimate for explicit dynamics analyses with solid-shell elements. Computers \& Structures 2013; 127:39 - 52. Special Issue IASS-IACM-2012.

7. Cocchetti G, Pagani M, Perego U. Selective mass scaling for distorted solid-shell elements in explicit dynamics: optimal scaling factor and stable time step estimate. International Journal for Numerical Methods in Engineering 2015; 101(9):700-731.

8. Frías G, Aquino W, Pierson K, Heinstein M, Spencer B. A multiscale mass scaling approach for explicit time integration using proper orthogonal decomposition. International Journal for Numerical Methods in Engineering 2014; 97(11):799-818.

9. Macek RW, Aubert BH. A mass penalty technique to control the critical time increment in explicit dynamic finite element analyses. Earthquake engineering \& structural dynamics 1995; 24(10):1315-1331.

10. Tkachuk A, Bischoff M. Local and global strategies for optimal selective mass scaling. Computational Mechanics 2014; 53(6):1197-1207.

11. Tkachuk A, Bischoff M. Direct and sparse construction of consistent inverse mass matrices: general variational

formulation and application to selective mass scaling. International Journal for Numerical Methods in Engineering $2015 ;$ 101(6):435-469.

12. Lombardo M, Askes H. Lumped mass finite element implementation of continuum theories with micro-inertia. International Journal for Numerical Methods in Engineering 2013; 96(7):448-466.

13. Lahiri SK, Bonet J, Peraire J, Casals L. A variationally consistent fractional time-step integration method for incompressible and nearly incompressible lagrangian dynamics. International journal for numerical methods in engineering 2005; 63(10):1371-1395.

14. Gil AJ, Lee CH, Bonet J, Aguirre M. A stabilised petrov-galerkin formulation for linear tetrahedral elements in compressible, nearly incompressible and truly incompressible fast dynamics. Computer Methods in Applied Mechanics and Engineering 2014; 276:659-690.

15. Lee CH, Gil AJ, Bonet J. Development of a stabilised petrov-galerkin formulation for conservation laws in lagrangian fast solid dynamics. Computer Methods in Applied Mechanics and Engineering 2014; 268:40-64.

16. Bonet J, Wood RD. Nonlinear continuum mechanics for finite element analysis. Cambridge university press, 1997.

17. Cho S, Park K, Huh H. A method for multidimensional wave propagation analysis via component-wise partition of longitudinal and shear waves. International Journal for Numerical Methods in Engineering 2013; 95(3):212-237.

18. Kolman R, Cho S, Park K. Efficient implementation of an explicit partitioned shear and longitudinal wave propagation algorithm. International Journal for Numerical Methods in Engineering 2015;

19. Schenk O, Gärtner K. Solving unsymmetric sparse systems of linear equations with pardiso. Future Generation Computer Systems 2004; 20(3):475-487.

20. Gennisson JL, Rénier M, Catheline S, Barrière C, Bercoff J, Tanter M, Fink M. Acoustoelasticity in soft solids: Assessment of the nonlinear shear modulus with the acoustic radiation force. The Journal of the Acoustical Society of America 2007; 122(6):3211-3219.

21. Destrade M, Ogden RW, Saccomandi G. Small amplitude waves and stability for a pre-stressed viscoelastic solid. Zeitschrift für angewandte Mathematik und Physik 2009; 60(3):511-528.

22. Palmeri ML, Sharma AC, Bouchard RR, Nightingale RW, Nightingale KR. A finite-element method model of soft tissue response to impulsive acoustic radiation force. Ultrasonics, Ferroelectrics and Frequency Control, IEEE

This article is protected by copyright. All rights reserved. 
Transactions on 2005; 52(10):1699-1712.

23. Holzapfel GA. Nonlinear solid mechanics, vol. 24. Wiley Chichester, 2000.

24. Olovsson L, Simonsson K. Iterative solution technique in selective mass scaling. Communications in numerical methods in engineering 2006; 22(1):77-82.
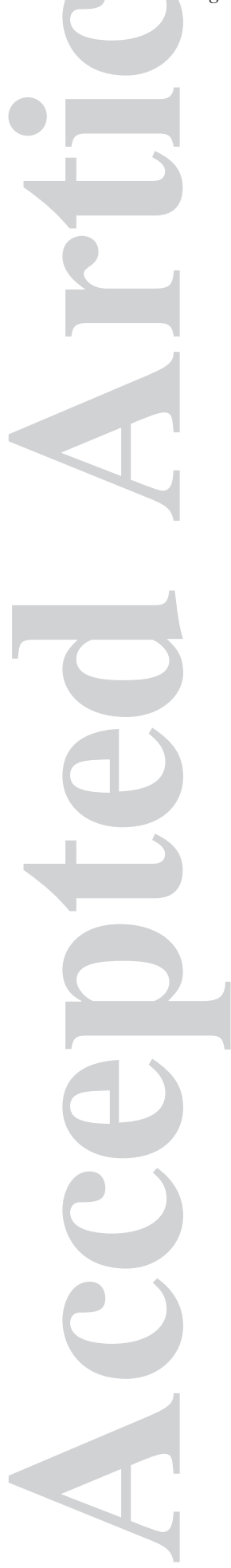

This article is protected by copyright. All rights reserved. 


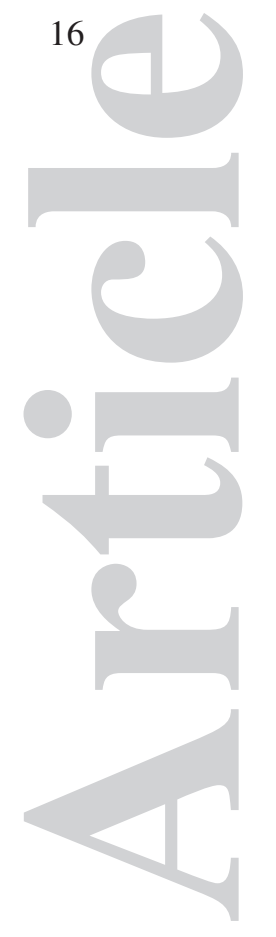

W. YE $E T A L$.

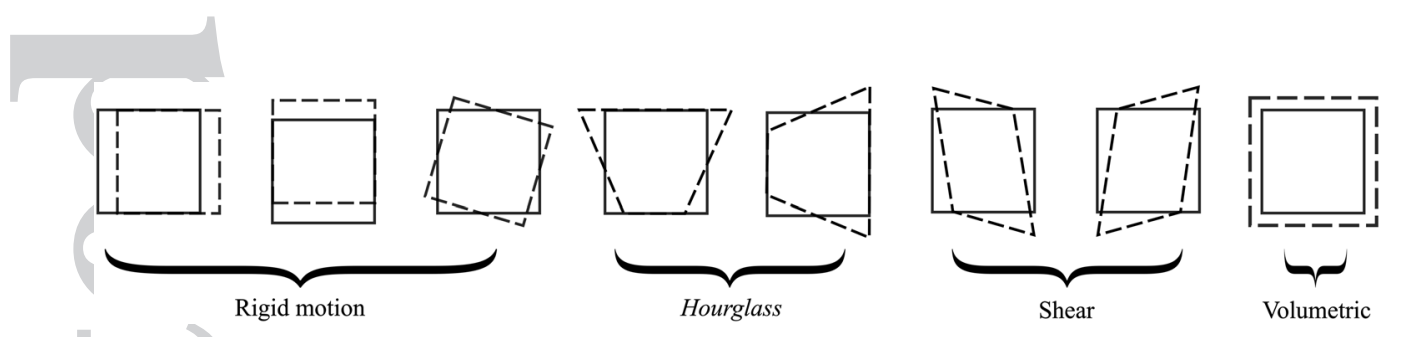

Figure 1. All eigenvectors of an ideal quadrilateral element (given by Tab.I)

This article is protected by copyright. All rights reserved. 

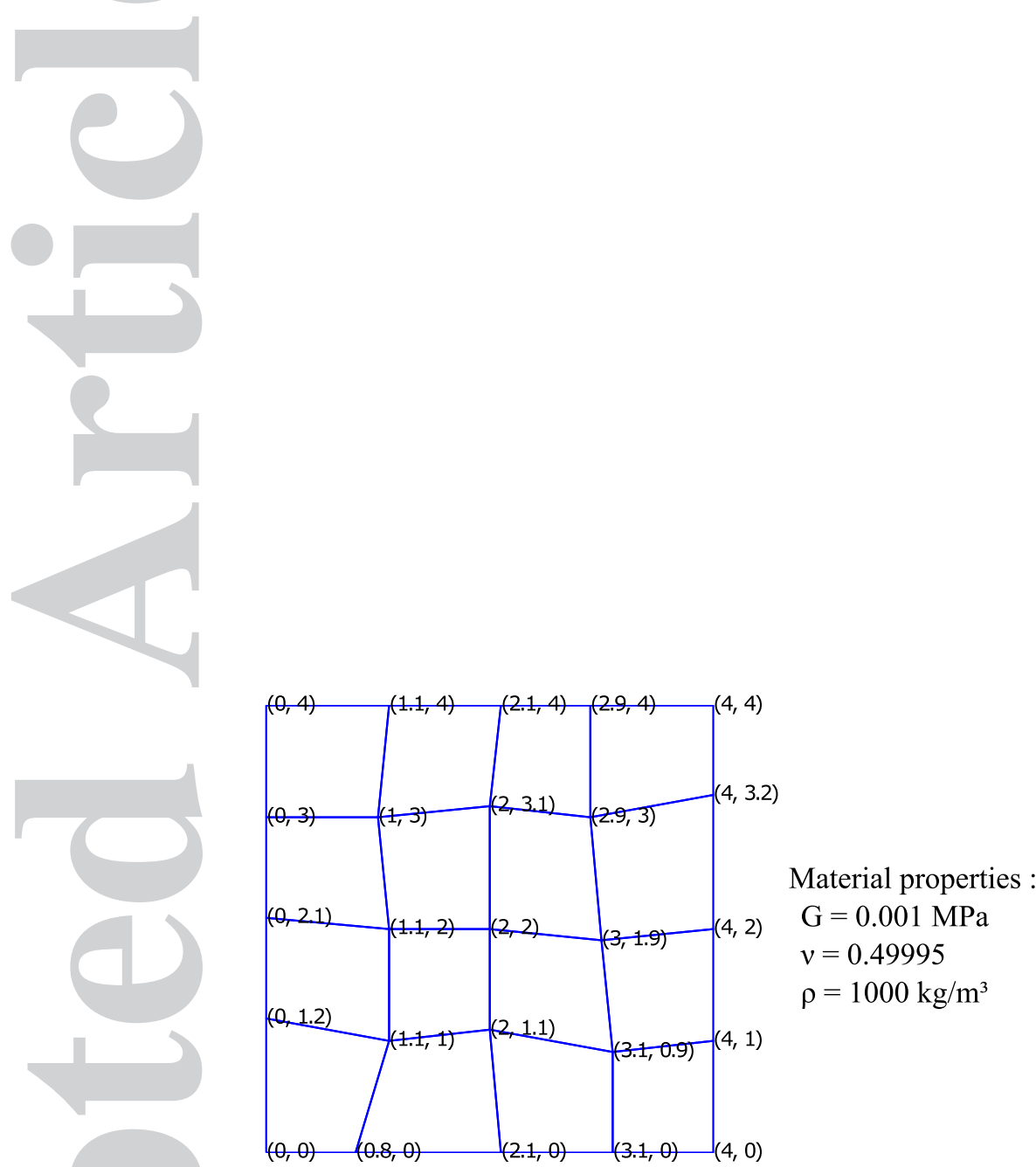

Figure 2. Test model: a square plate with distorted mesh of 50 degrees of freedom.

This article is protected by copyright. All rights reserved. 


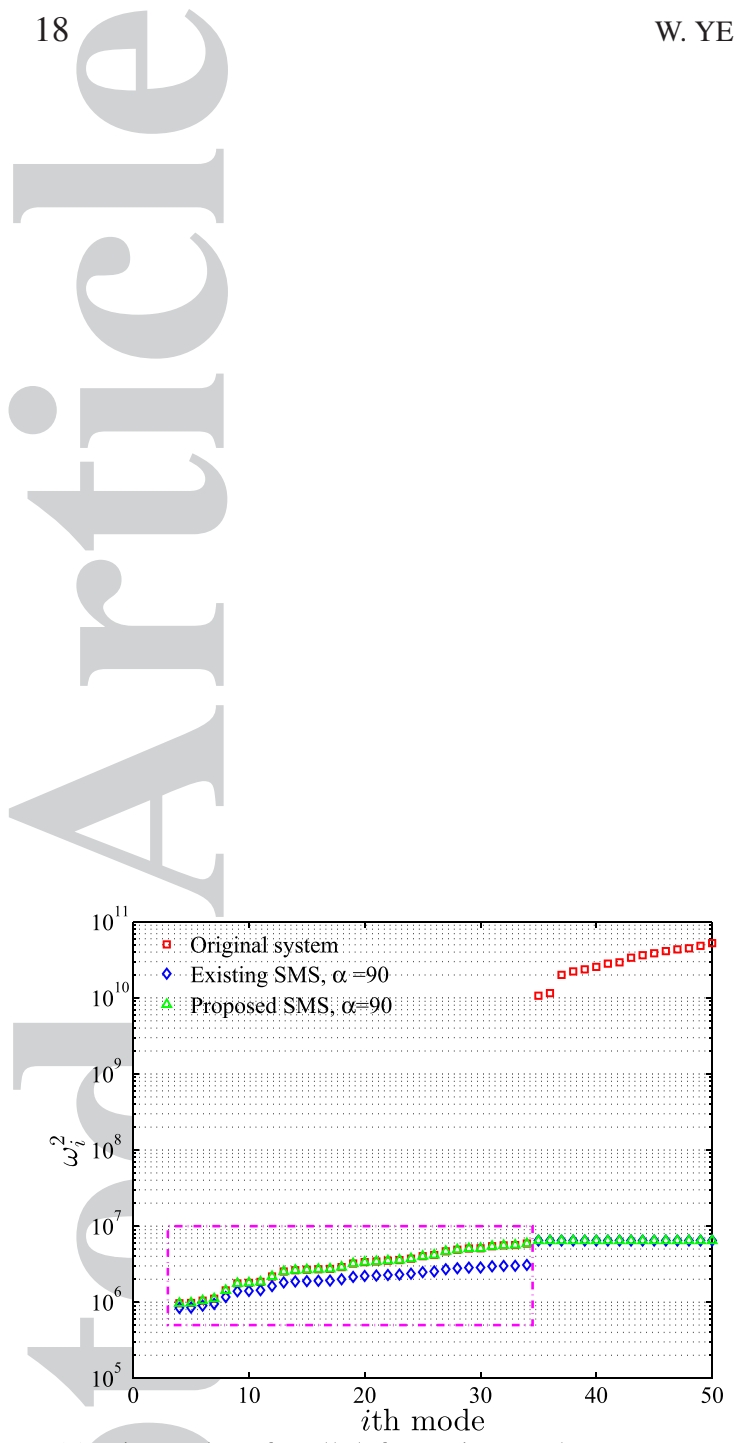

(a) Eigenvalues for all deformation modes

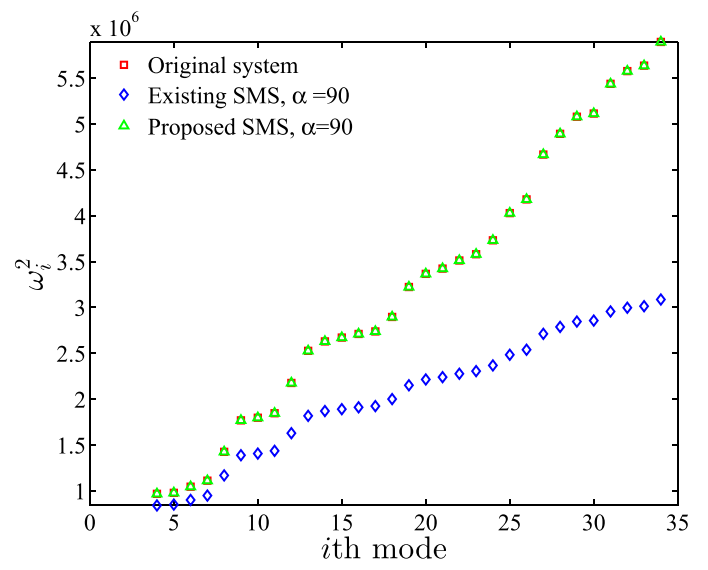

(b) Eigenvalues for shear deformation modes (zoom of left figure)

Figure 3. Eigenvalues of test model, first three (rigid motion) modes absent

This article is protected by copyright. All rights reserved. 


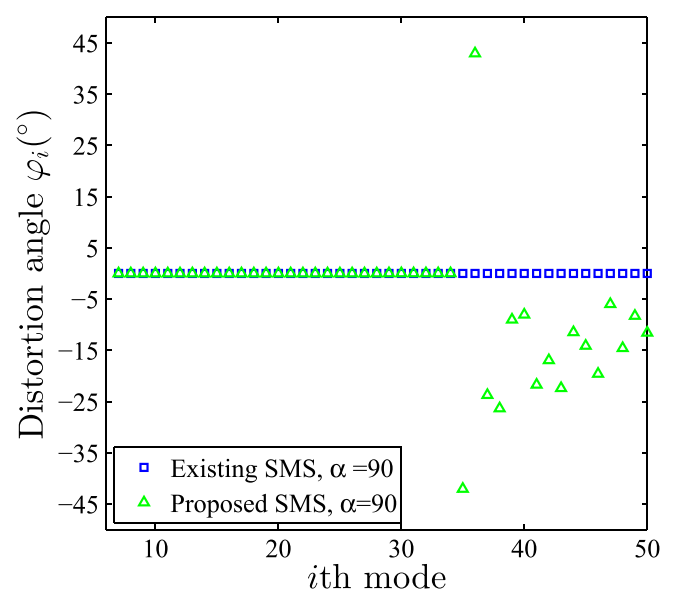

Figure 4. Eigenvectors distortion of test model (first three rigid body modes absent)

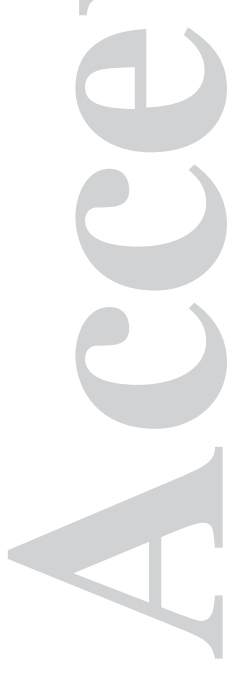

This article is protected by copyright. All rights reserved. 


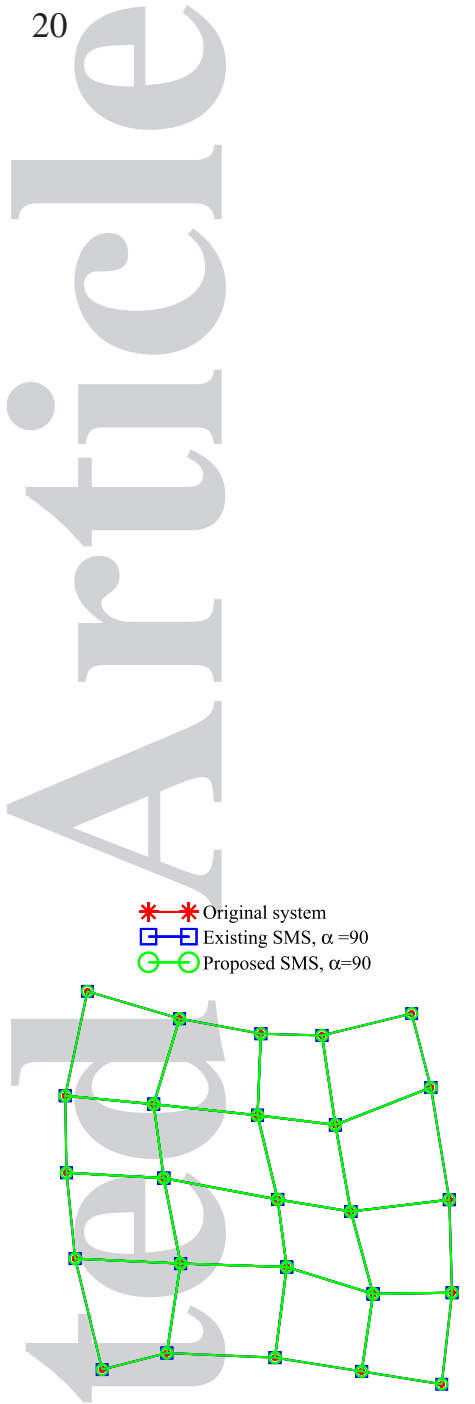

(a) 4th eigenmode: first shear mode

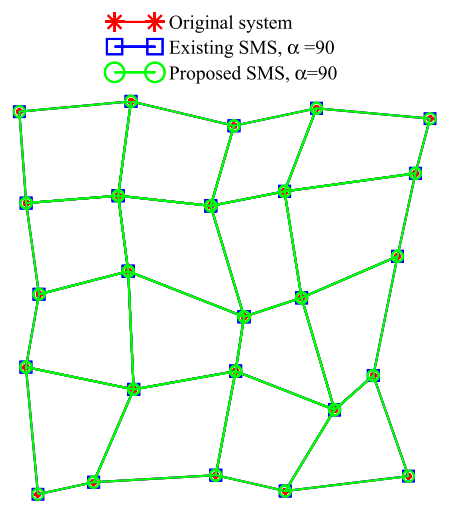

(b) 21th eigenmode, intermediate shear mode

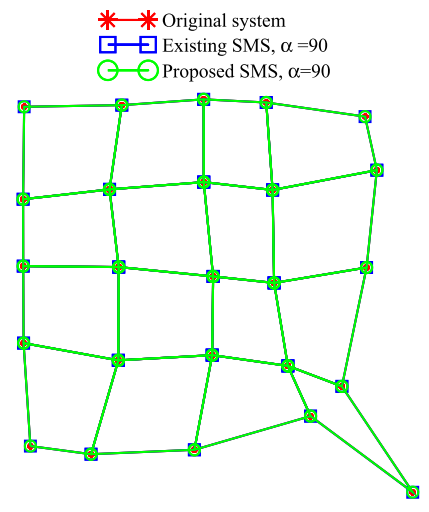

(c) 34th eigenmode, last shear mode

Figure 5. Deformations of eigenvectors for 3 shear eigenmode

This article is protected by copyright. All rights reserved. 


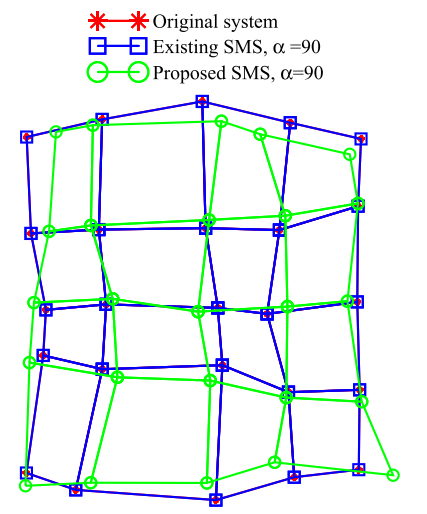

(b) 43th eigenmode, intermediate volumetric mode

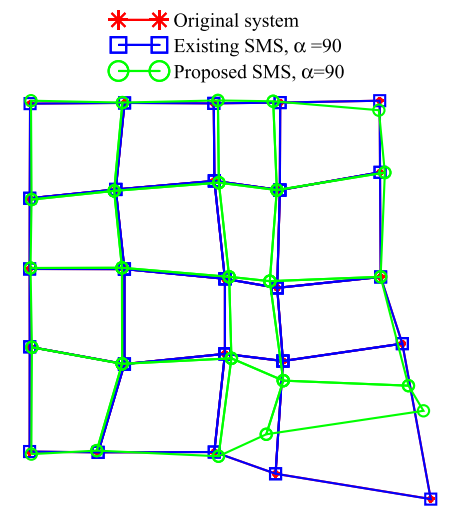

(c) 50th eigenmode, last volumetric mode (a) 35th eigenmode, first volumetric mode

Figure 6. Deformations of eigenvectors for 3 volumetric eigenmode

This article is protected by copyright. All rights reserved. 


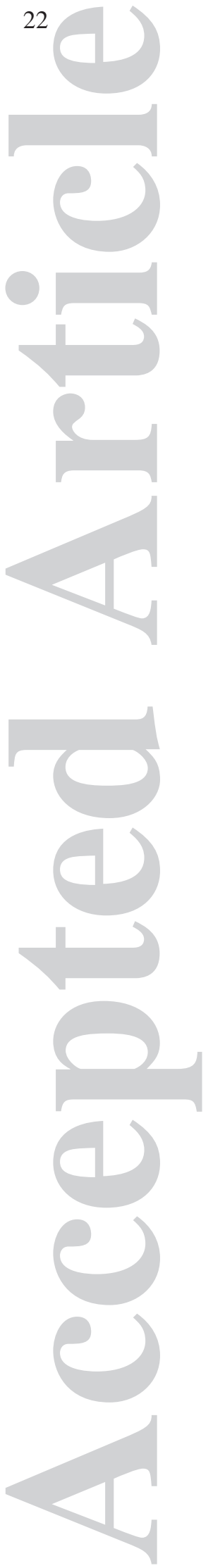

W. YE ET AL.

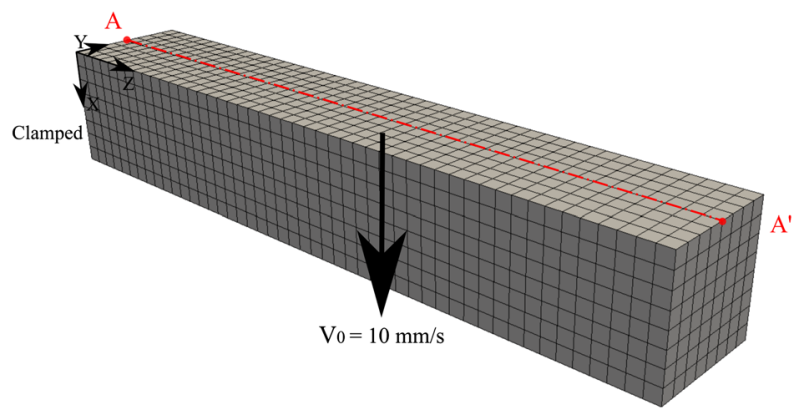

Figure 7. Cantilever beam model, dimension: $1 \times 1 \times 6 \mathrm{~mm}$.

This article is protected by copyright. All rights reserved. 

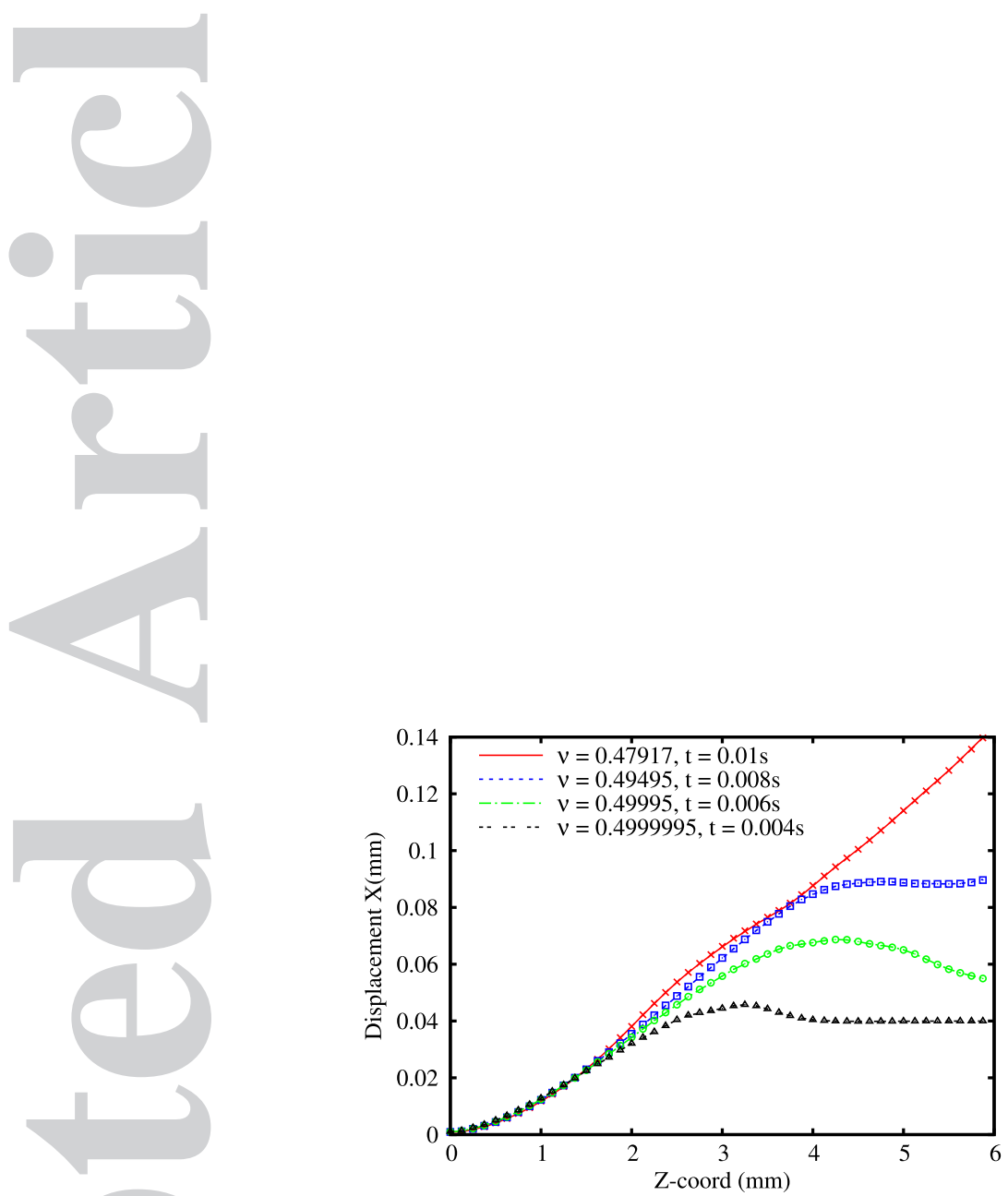

Figure 8. Cantilever Beam: Displacement along A-A' for different cases. Lines: results by pure explicit simulation; Marks: results by proposed SMS method

This article is protected by copyright. All rights reserved. 


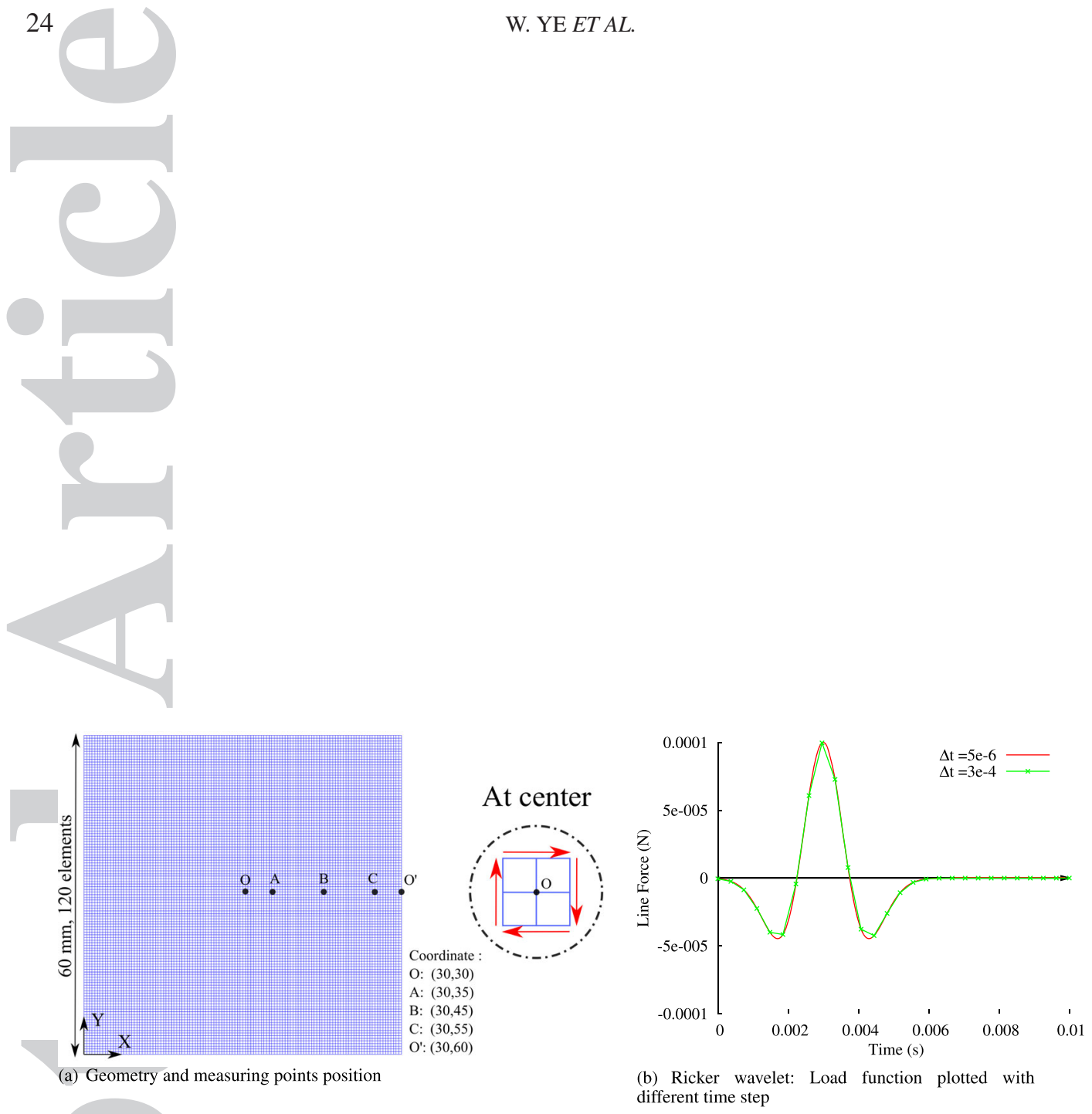

Figure 9. Circular shear wave propagation model

This article is protected by copyright. All rights reserved. 

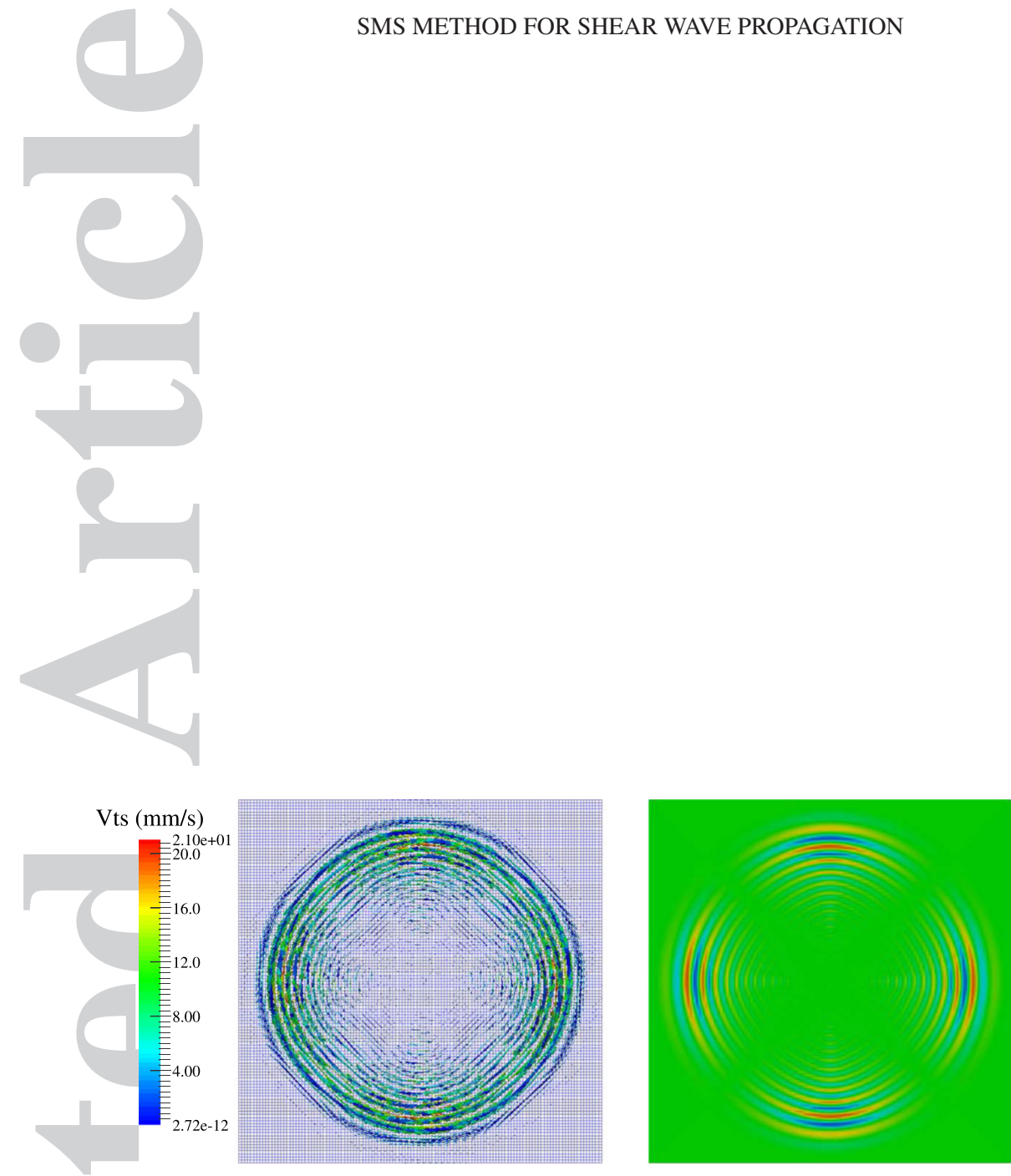

s12 (MPa)

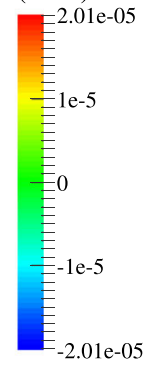

Figure 10. Circular shear wave propagation: Velocity distribution (Left) and shear stress distribution (Right) at $t=0.03 \mathrm{~s}$. Results obtained by proposed SMS method with $\alpha=30$.

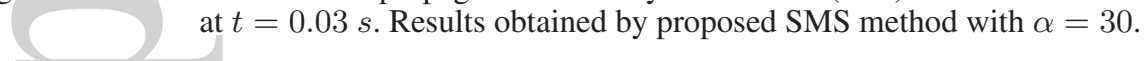

This article is protected by copyright. All rights reserved. 


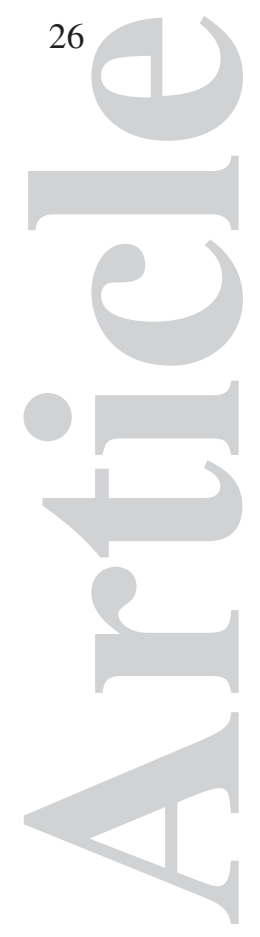

W. YE $E T A L$.
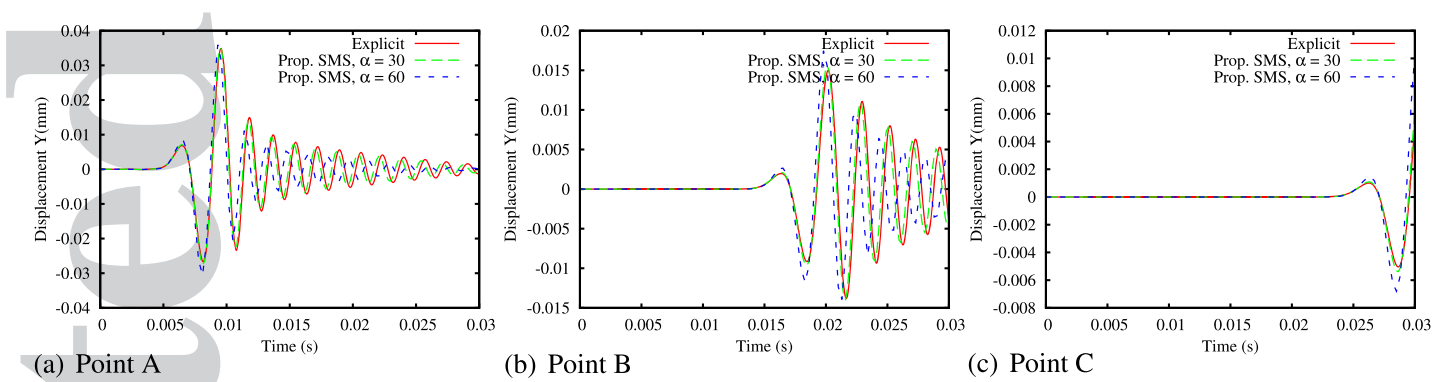

(c) Point C

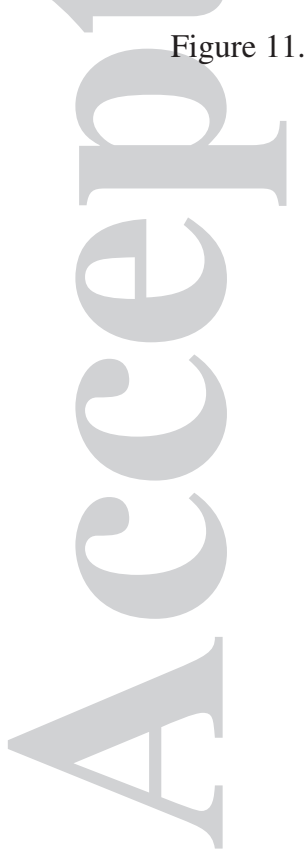

This article is protected by copyright. All rights reserved. 


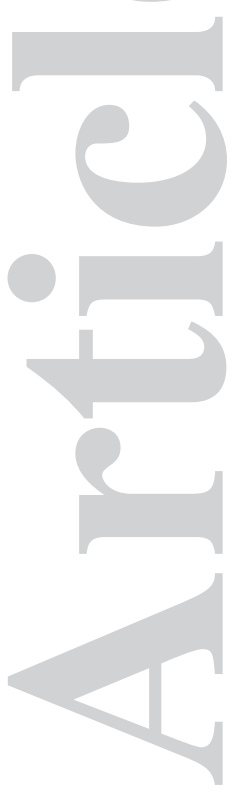

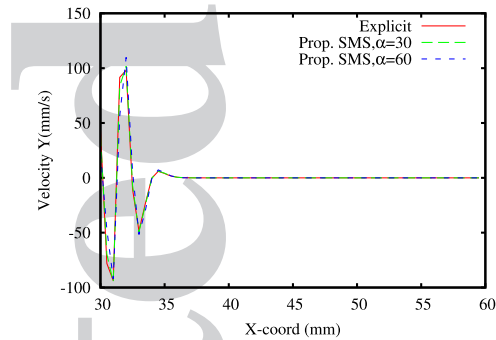

(a) $t=0.006 \mathrm{~s}$

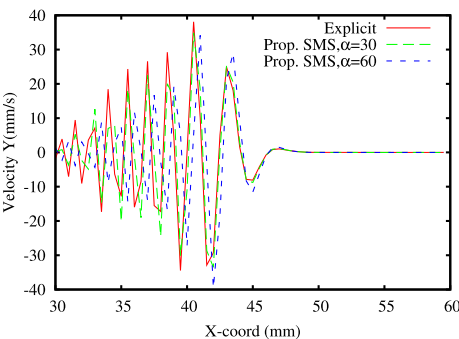

(b) $t=0.018 \mathrm{~s}$

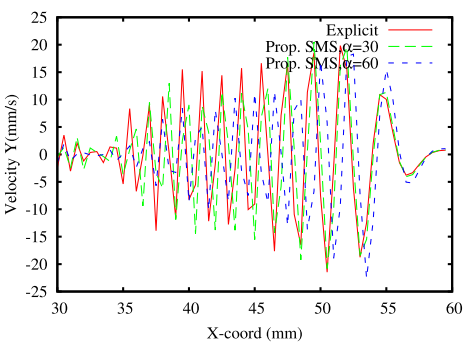

(c) $t=0.03 \mathrm{~s}$

Figure 12. Circular shear wave propagation: Vertical velocity along O-O’ at different time

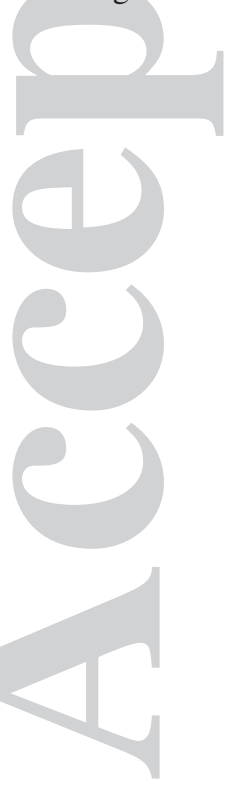

This article is protected by copyright. All rights reserved. 


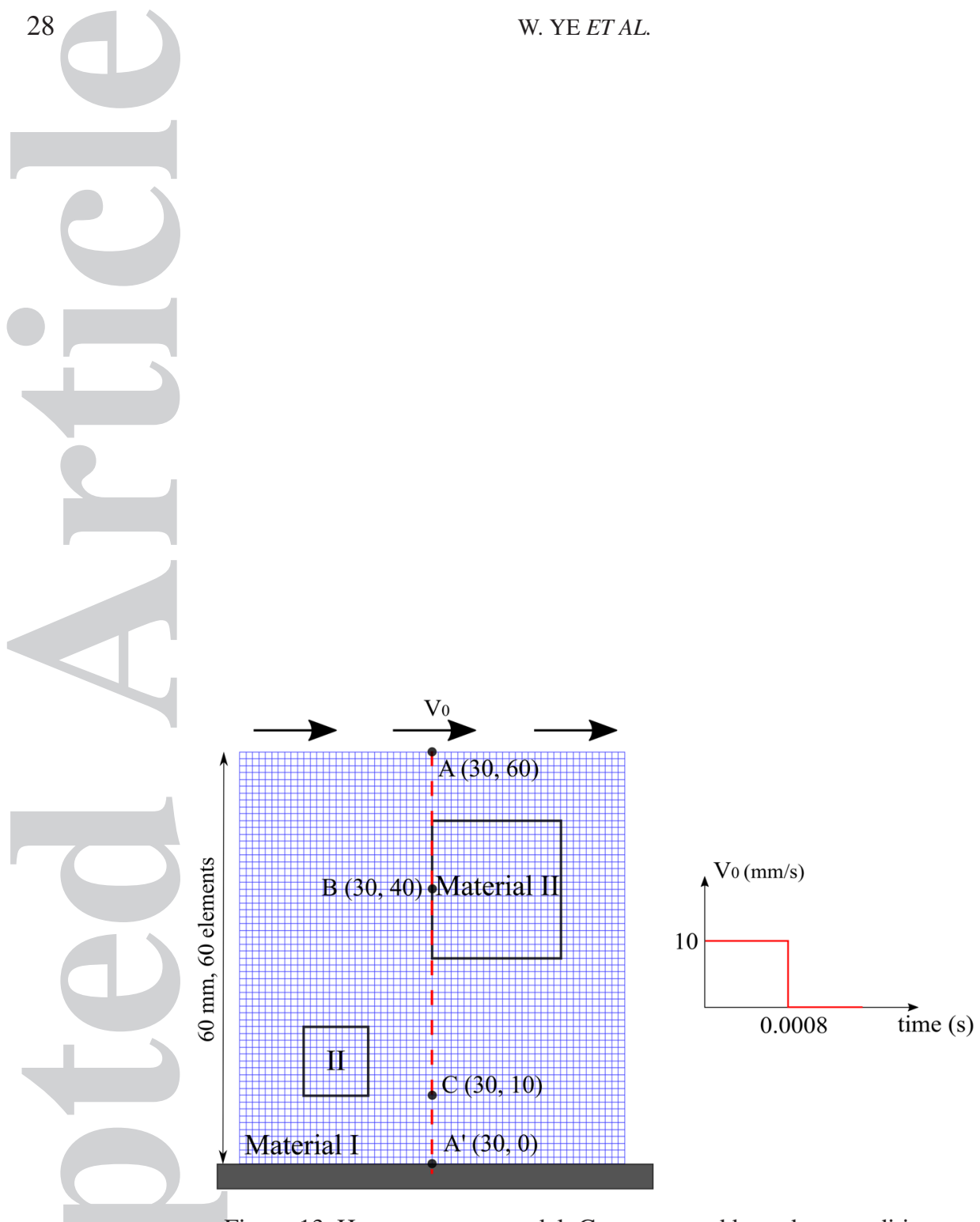

Figure 13. Heterogeneous model: Geometry and boundary conditions

This article is protected by copyright. All rights reserved. 

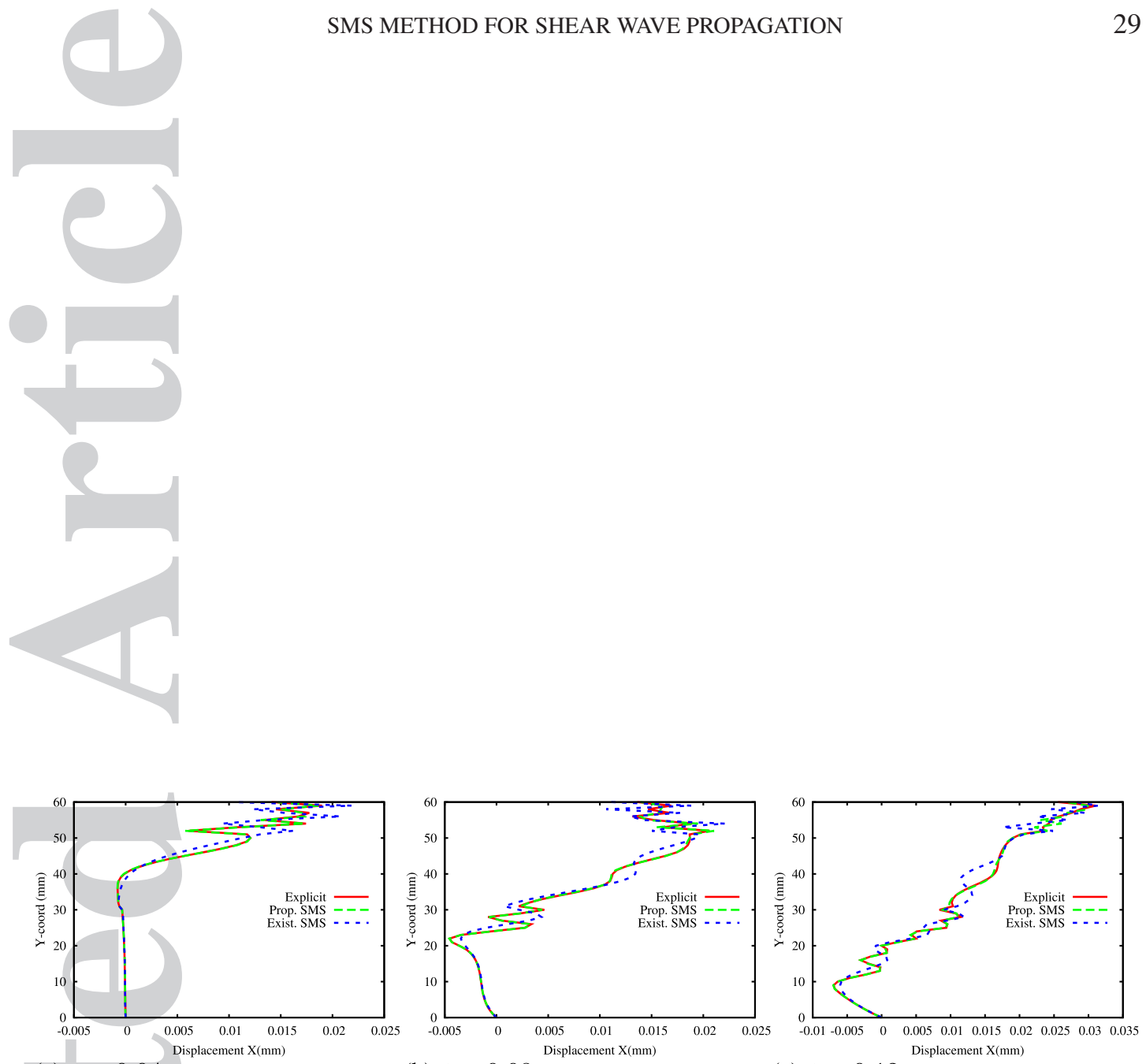

(a) $t=0.04 \mathrm{~s}$

(b) $t=0.08 \mathrm{~s}$

(c) $t=0.12 \mathrm{~s}$

Figure 14. Heterogeneous model: Horizontal displacement along A-A' at different time

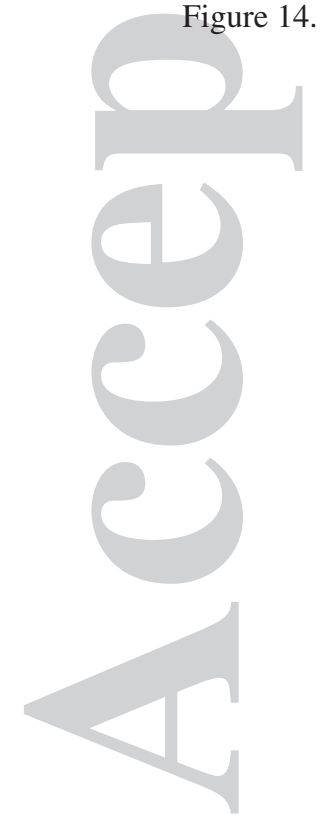

This article is protected by copyright. All rights reserved. 


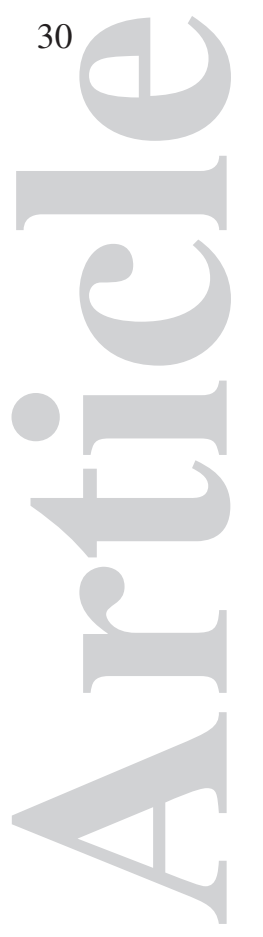

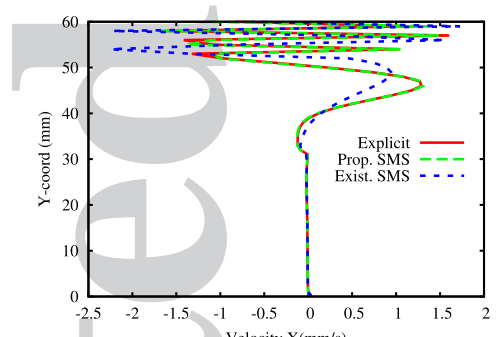

(a) $t=0.04 \mathrm{~s}$

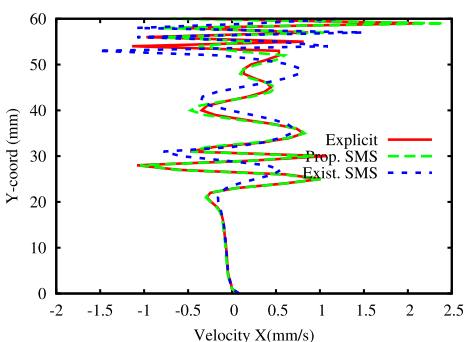

(b) $t=0.08 \mathrm{~s}$

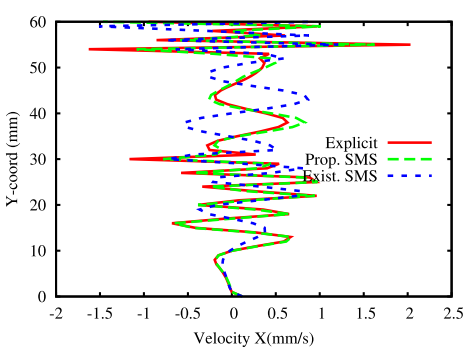

(c) $t=0.12 \mathrm{~s}$

Figure 15. Heterogeneous model: Horizontal velocity along A-A' at different time

This article is protected by copyright. All rights reserved. 


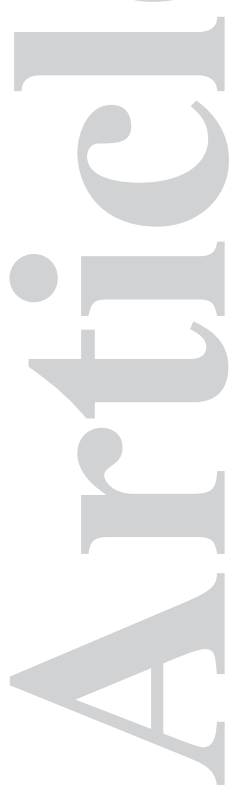

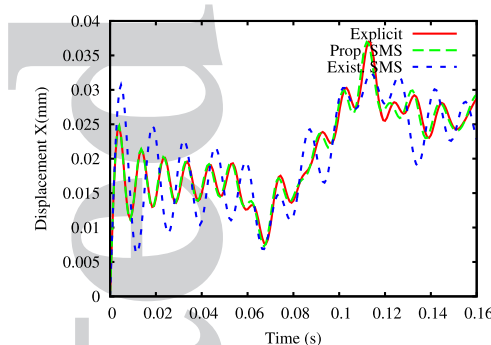

(a) Point A

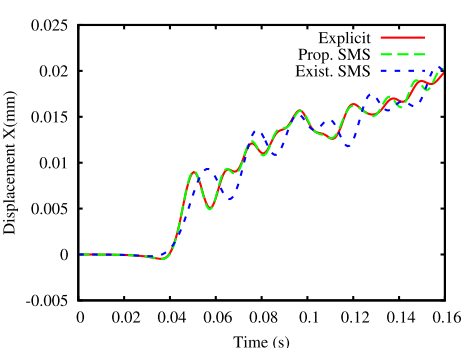

(b) Point B

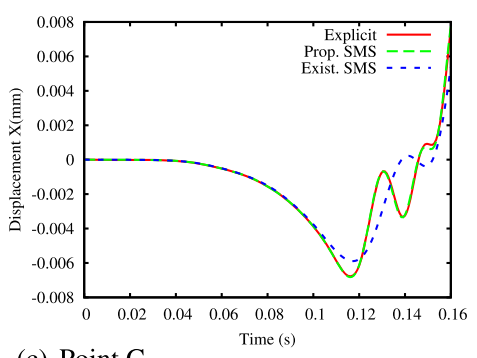

(c) Point C

Figure 16. Heterogeneous model: Horizontal displacement at measuring points

This article is protected by copyright. All rights reserved. 


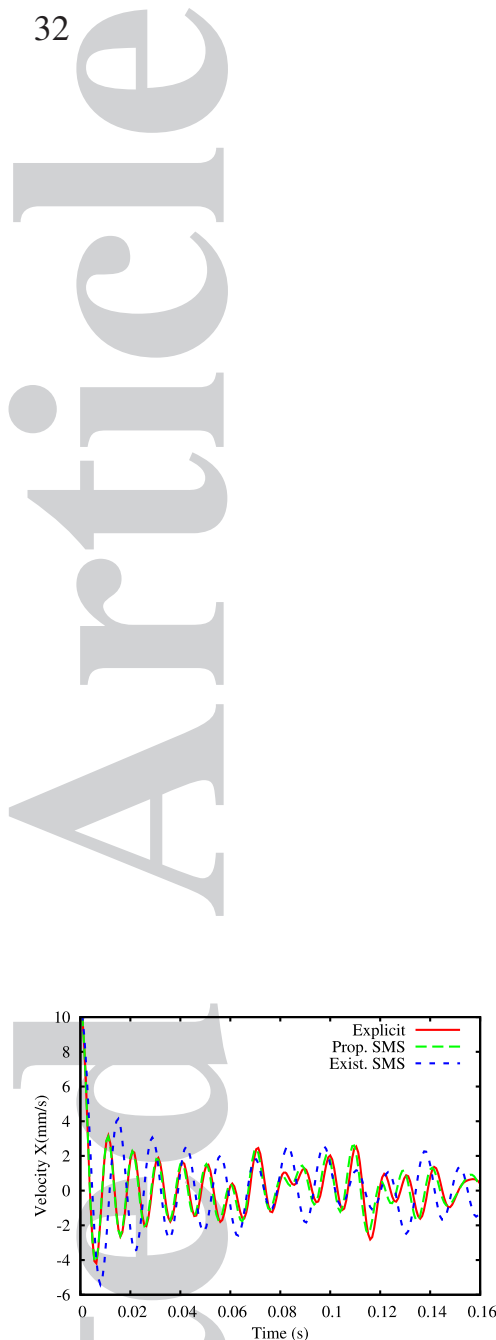

(a) Point $\mathrm{A}$

W. YE $E T A L$.

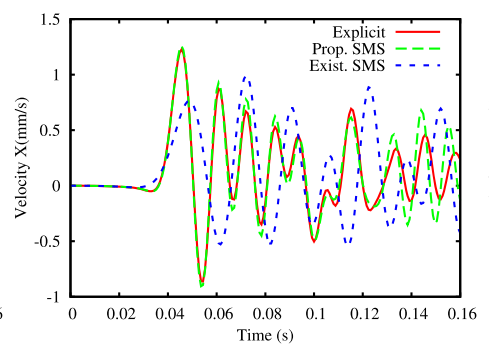

(b) Point $\mathrm{B}$

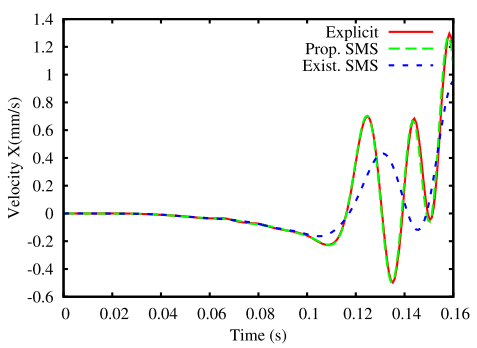

(c) Point $\mathrm{C}$

Figure 17. Heterogeneous model: Horizontal velocity at measuring points

This article is protected by copyright. All rights reserved. 


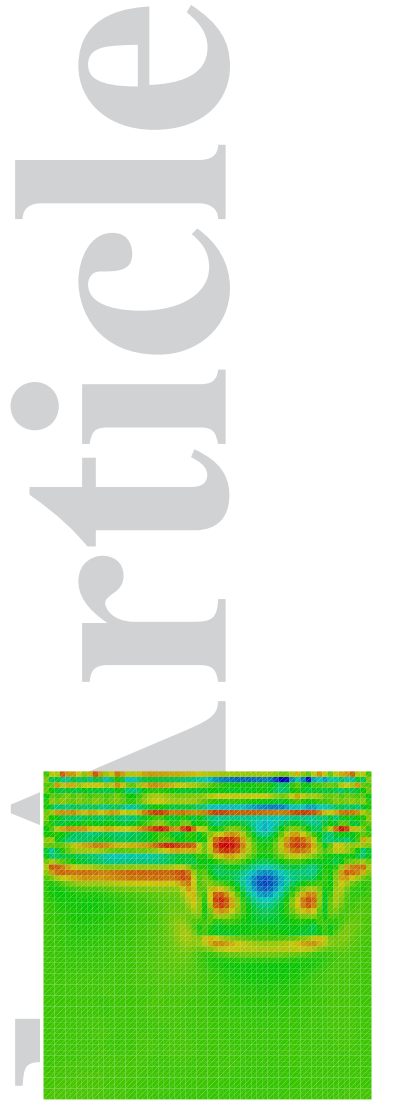

(a) at $t=0.064 \mathrm{~s}$

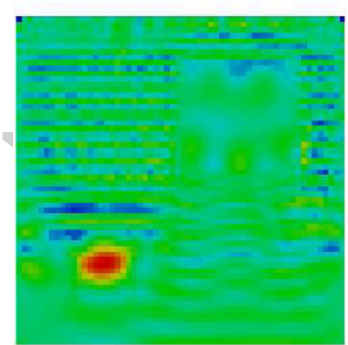

(b) at $t=0.16 \mathrm{~s}$
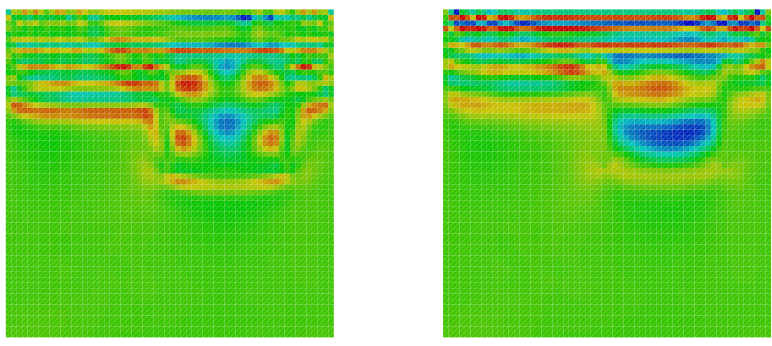

s12 (MPa)
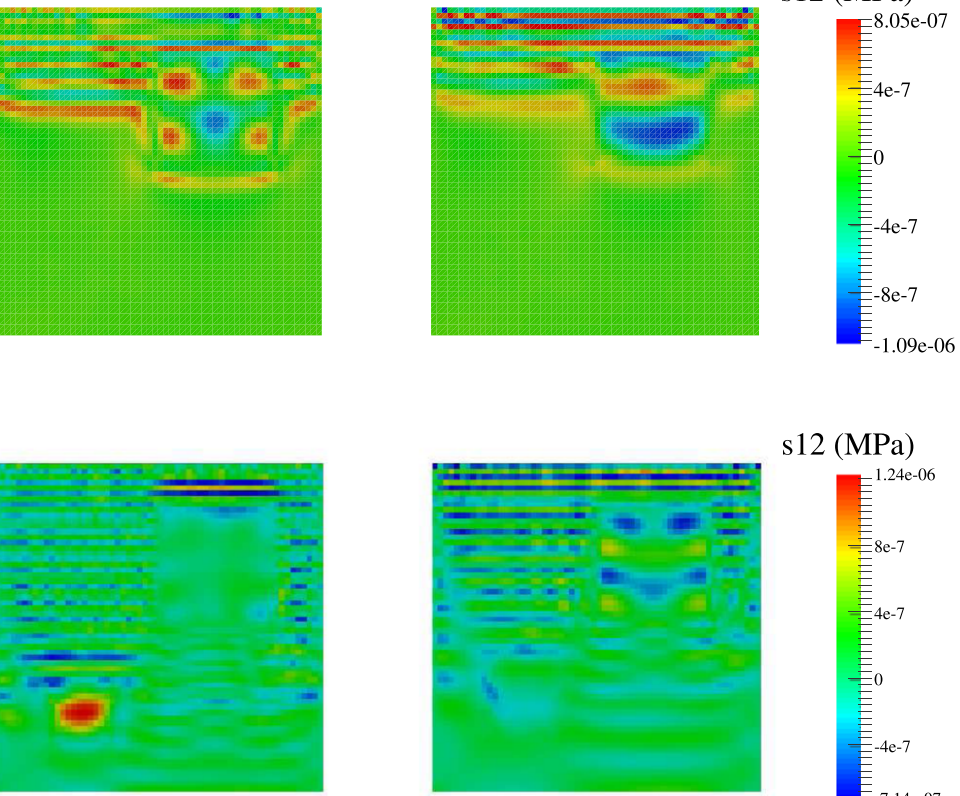

s12 (MPa)

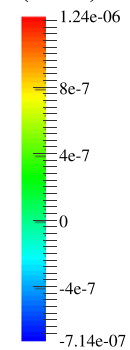

Figure 18. Heterogeneous model: Shear stress distribution. Left: Pure explicit; Middle: Proposed SMS; Right: Existing SMS

This article is protected by copyright. All rights reserved. 


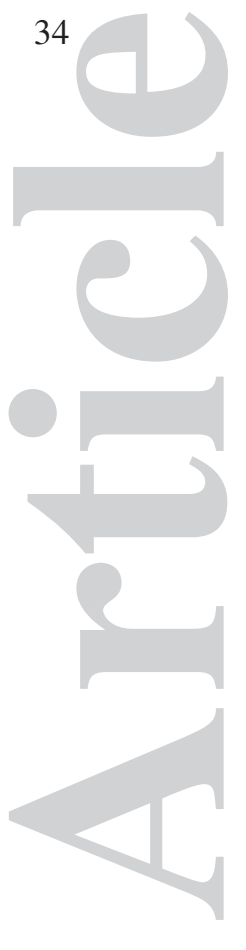

W. YE ET AL.
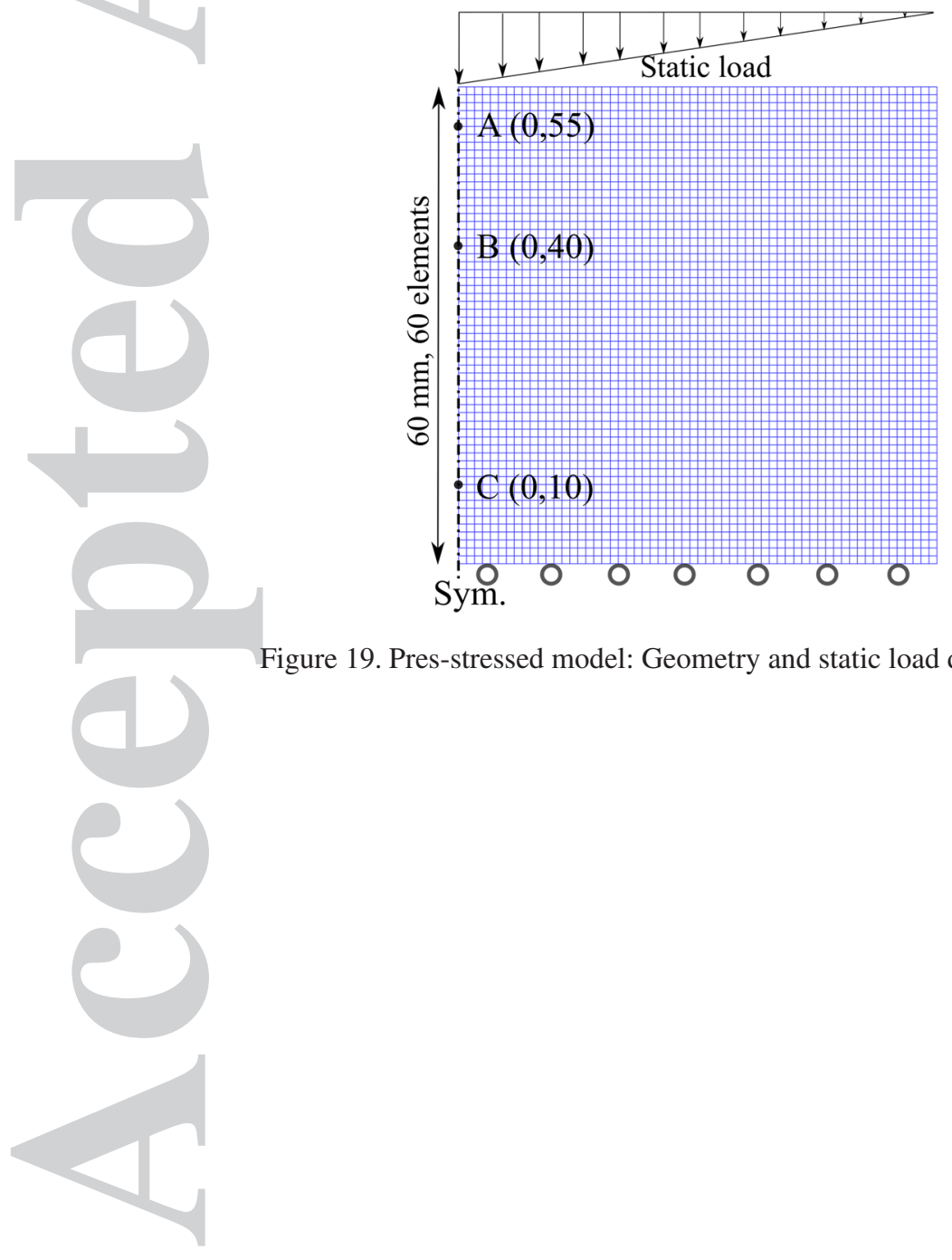

Figure 19. Pres-stressed model: Geometry and static load distribution

This article is protected by copyright. All rights reserved. 

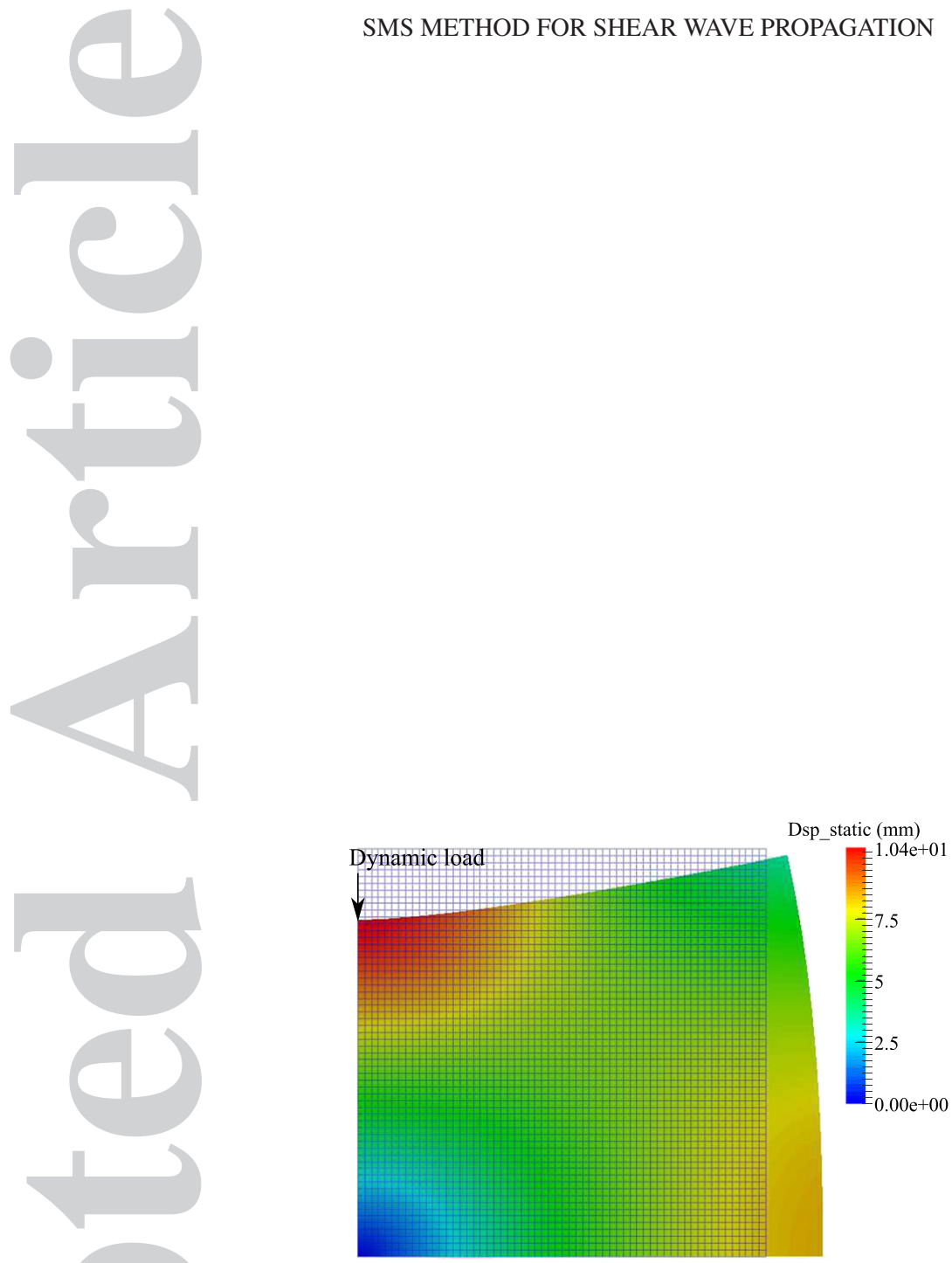

Figure 20. Deformed configuration after static load, amplification factor $=1$

This article is protected by copyright. All rights reserved. 


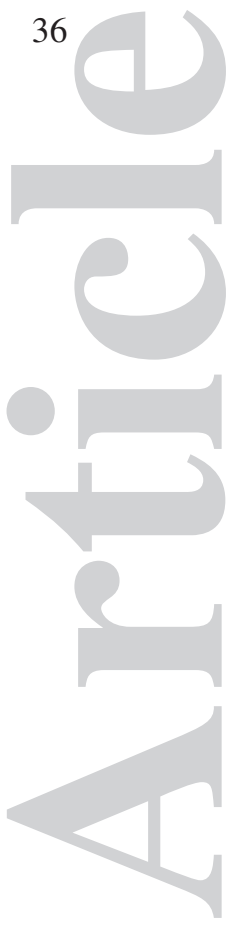

W. YE ET AL.
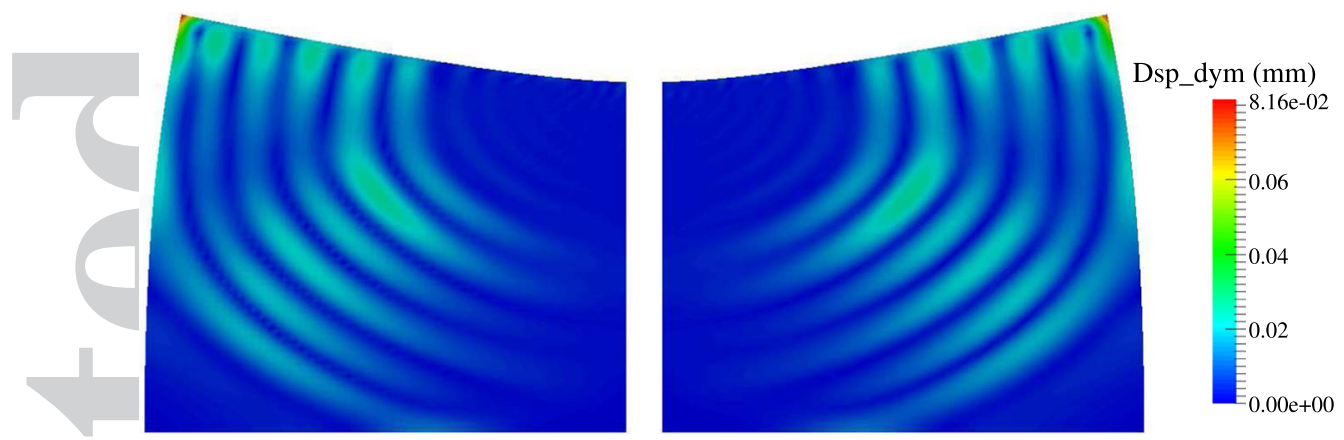

Figure 21. Dynamic displacement distribution at $t=0.05 \mathrm{~s}$. Left: Explicit; Right: Proposed SMS.
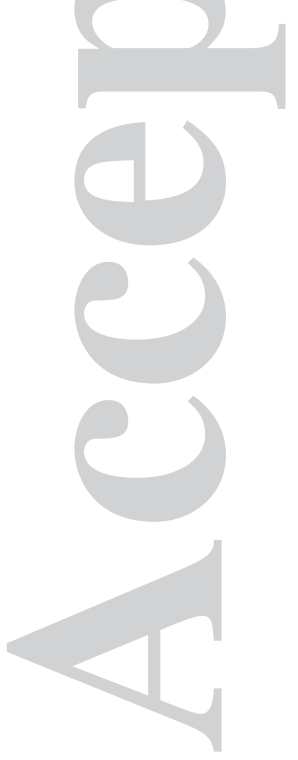

This article is protected by copyright. All rights reserved. 


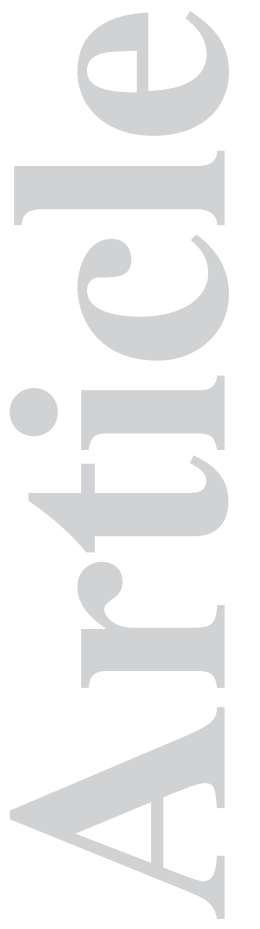

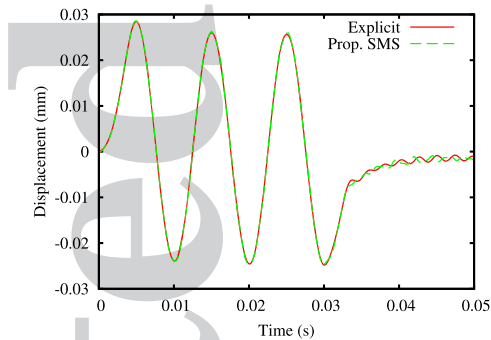

(a) Point A

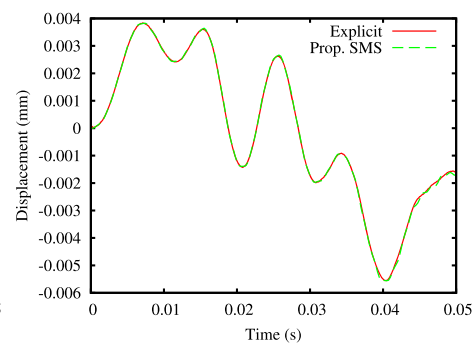

(b) Point B

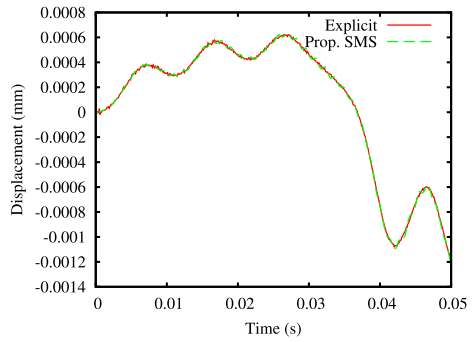

(c) Point C

Figure 22. Pre-stressed model: Vertical displacement at measuring points

This article is protected by copyright. All rights reserved. 


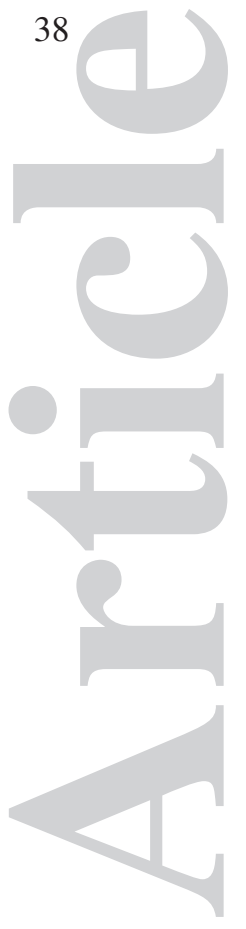

W. YE $E T A L$.

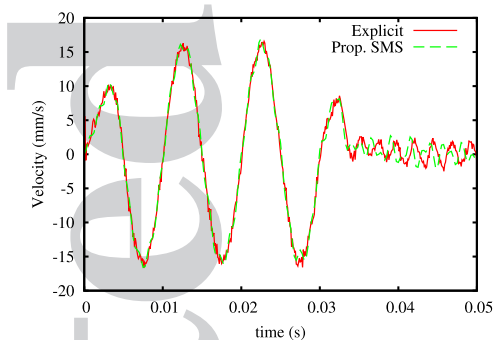

(a) Point $\mathrm{A}$

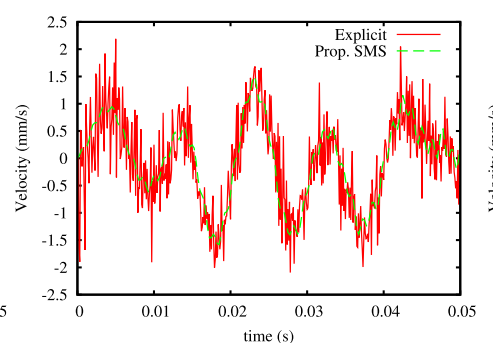

(b) Point B

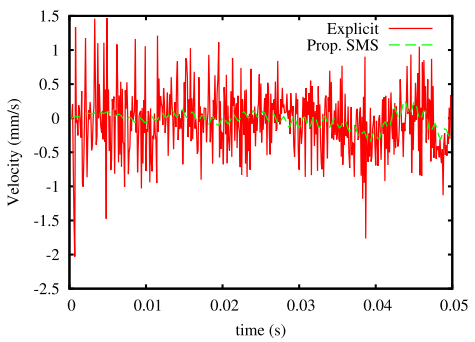

(c) Point C

Figure 23. Pre-stressed model: Vertical velocity at measuring points

This article is protected by copyright. All rights reserved. 

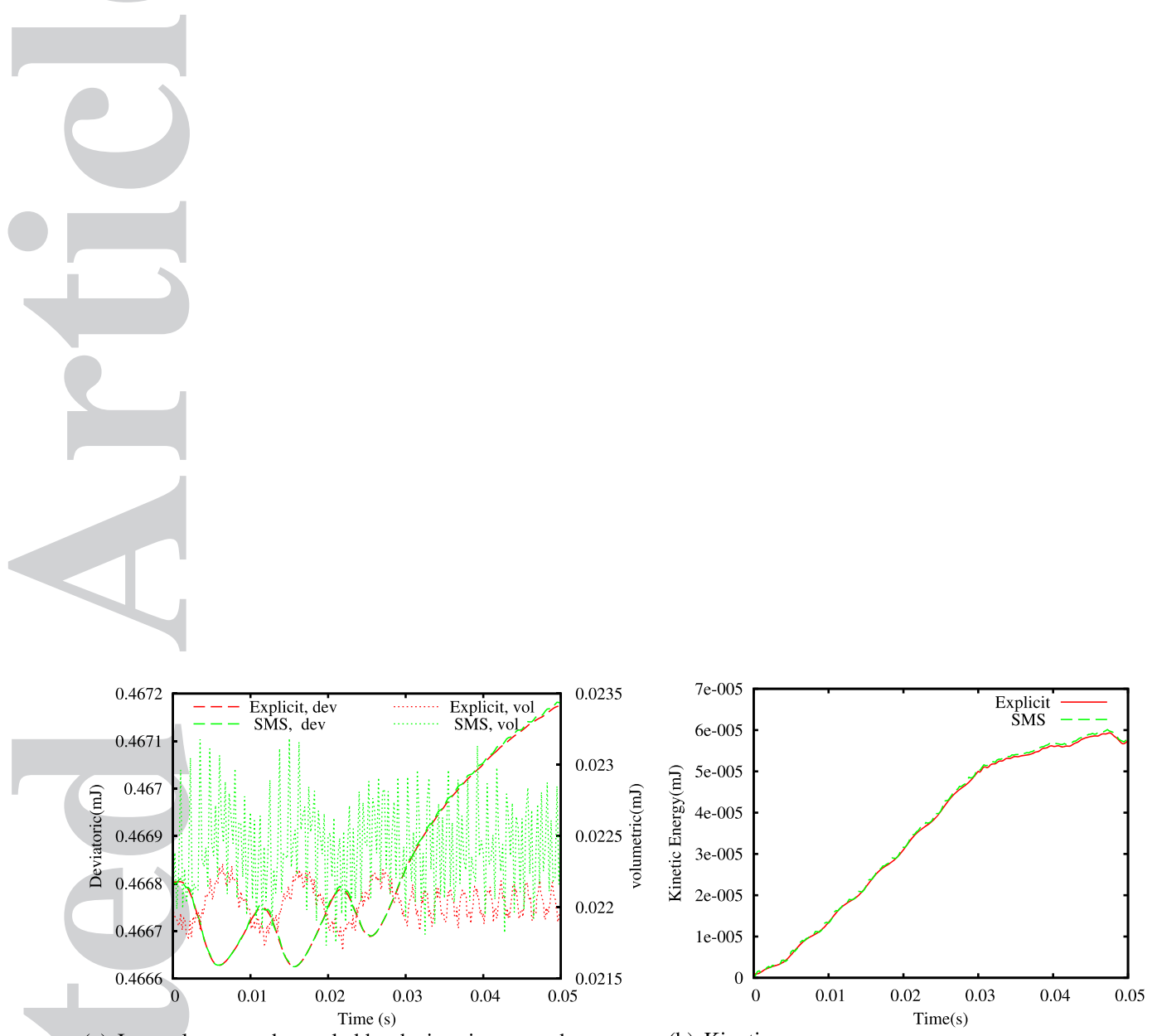

(a) Internal energy, decoupled by deviatoric part and volumetric part

(b) Kinetic energy

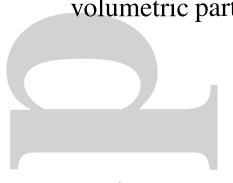

Figure 24. Pre-stressed model: Time history of energy evolution

This article is protected by copyright. All rights reserved. 


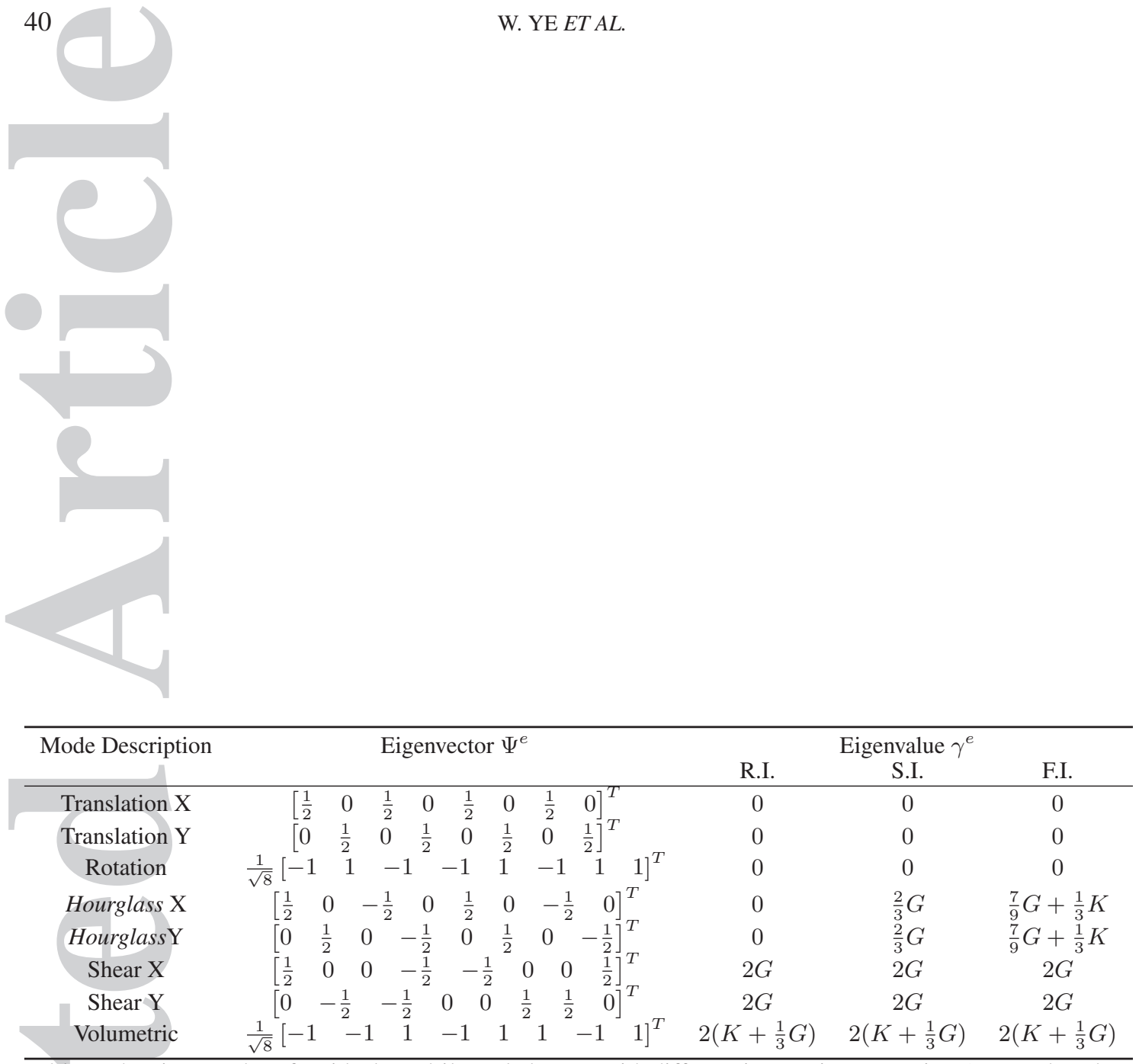

Table I. The eigenmodes of an ideal quadrilateral element with different integration strategies. (R.I.: reduced integration; S.I.: selective integration; F.I.: full integration;)

This article is protected by copyright. All rights reserved. 


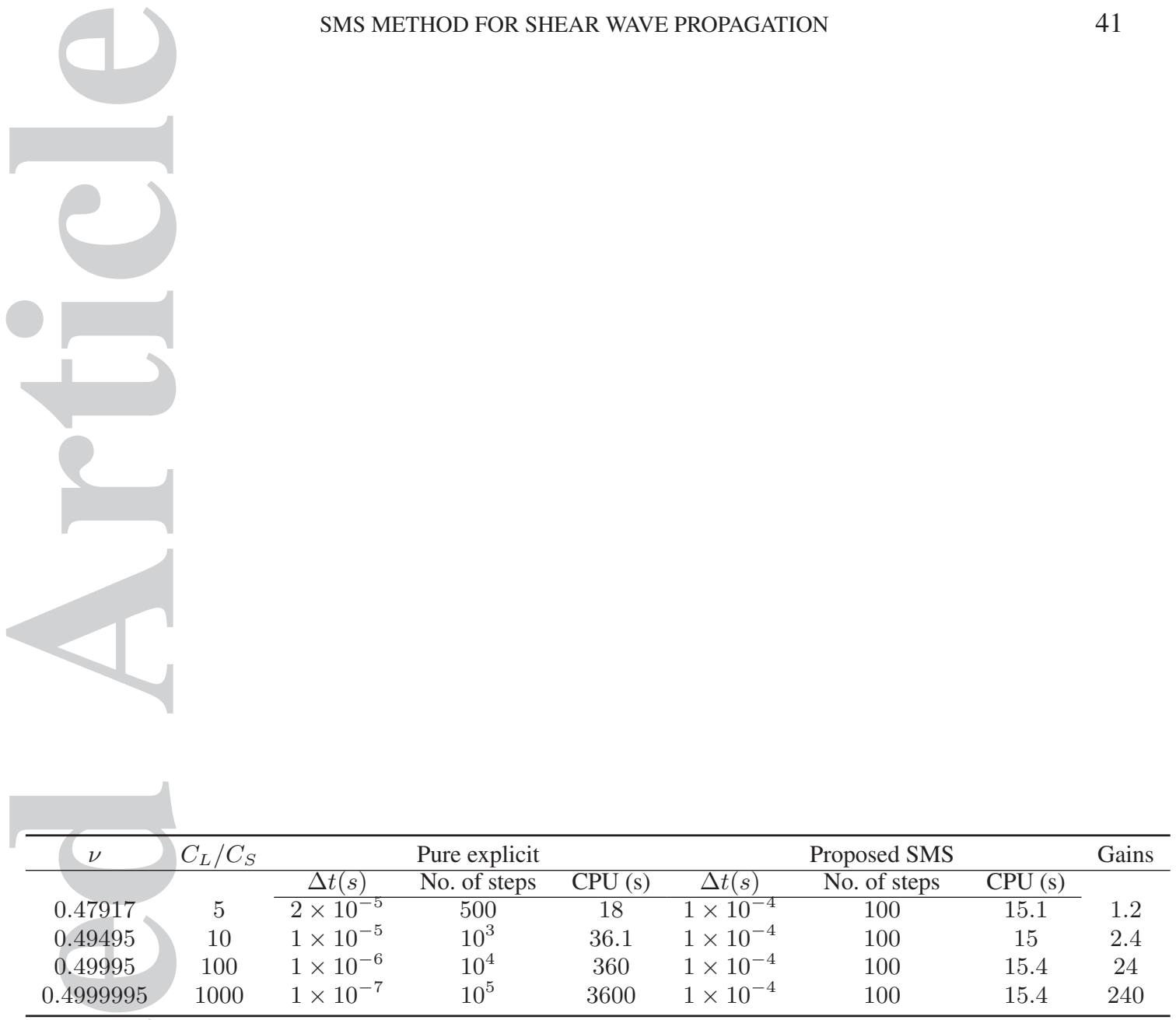

Table II. Cantilever beam model: Summary of simulation parameters

This article is protected by copyright. All rights reserved. 


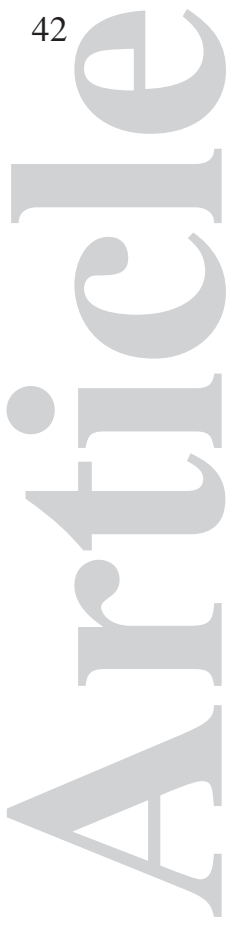

W. YE $E T A L$.

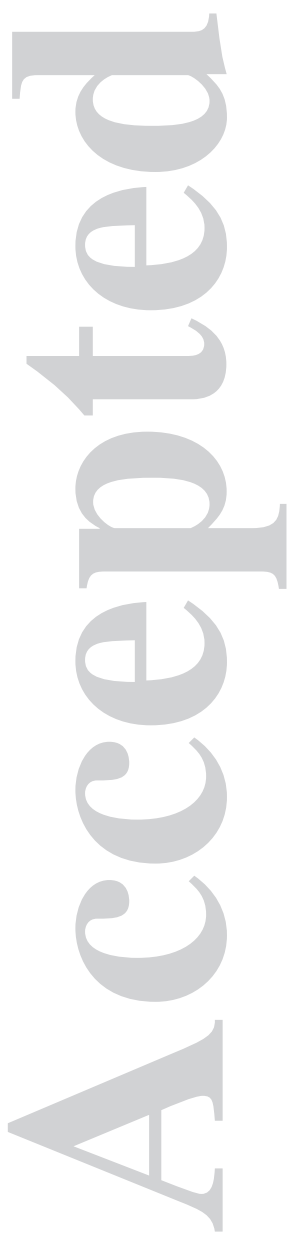

\begin{tabular}{ccc}
\hline & Material I & Material II \\
\hline$\rho\left(k g / m^{3}\right)$ & 1000 & 1000 \\
$G(M P a)$ & $10^{-4}$ & $10^{-3}$ \\
$\nu$ & 0.49999 & 0.4 \\
$C_{L}(\mathrm{~m} / \mathrm{s})$ & 100 & 2.45 \\
$C_{S}(\mathrm{~m} / \mathrm{s})$ & 0.317 & 1 \\
\hline
\end{tabular}

Table III. Heterogeneous model: Material parameters

This article is protected by copyright. All rights reserved. 